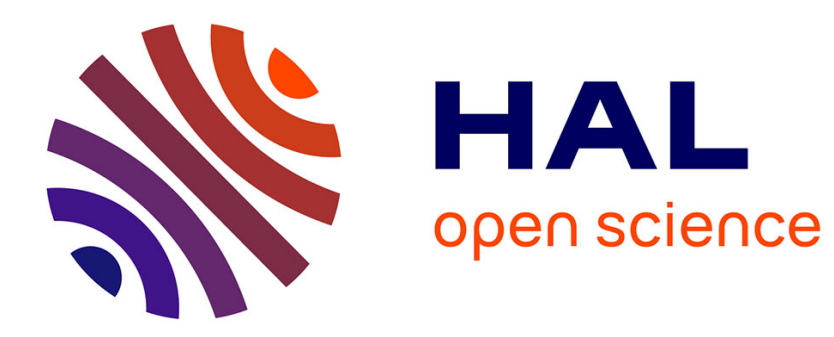

\title{
Un dragon pour emblème - Variations sur le titre du Wenxin diaolong
}

\author{
Valérie Lavoix
}

\section{To cite this version:}

Valérie Lavoix. Un dragon pour emblème - Variations sur le titre du Wenxin diaolong. Études Chinoises, 2000. hal-01695523

\section{HAL Id: hal-01695523 \\ https://hal.science/hal-01695523}

Submitted on 29 Jan 2018

HAL is a multi-disciplinary open access archive for the deposit and dissemination of scientific research documents, whether they are published or not. The documents may come from teaching and research institutions in France or abroad, or from public or private research centers.
L'archive ouverte pluridisciplinaire HAL, est destinée au dépôt et à la diffusion de documents scientifiques de niveau recherche, publiés ou non, émanant des établissements d'enseignement et de recherche français ou étrangers, des laboratoires publics ou privés. 


\title{
Un dragon pour emblème
}

\section{Variations sur le titre du Wenxin diaolong}

\author{
Valérie Lavoix ${ }^{1}$
}

Le plus célèbre traité de critique littéraire chinois, composé vers l'an 500 par Liu Xie (ca. 465-ca. 521), homme de lettres dont la renommée auprès de ses contemporains fut paradoxalement bien plus grande en tant qu'écrivain et bibliographe bouddhiste ${ }^{2}$, porte ce qui est sans doute l'un des titres les mieux réussis de la littérature ancienne. Les quatre mots qui le composent affichent en évidence, à la fois comme programme et métaphore, l'ambition de l'auteur en tant que critique et littérateur, mais ce au prix d'une figure à la fois subtile et quelque peu retorse : les longues syllabes

1 Valérie Lavoix est Maître de conférences à l' INALCO, Centre Dauphine, place du Maréchal-de-Lattre-de-Tassigny, 75116 Paris. Cette étude est issue du chapitre VII d'une thèse de doctorat intitulée Liu Xie (ca. 465-ca. 521). Homme de lettres, bouddhiste laïc et juge des poètes, préparée avec le concours de la Chiang Ching-kuo Foundation for International Scholarly Exchange, sous la direction de Jacques Pimpaneau et François Martin, INALCO, 1997. Elle fut dès l'origine inspirée, faut-il le préciser, par la lecture du bel ouvrage de JeanPierre Diény : Le symbolisme du dragon dans la Chine antique, Paris, Collège de France, Institut des Hautes Études Chinoises, 1987 (« Bibliothèque de l'Institut des Hautes Études Chinoises », XXVII).

2 Cf. la biographie de Liu Xie dans le Liang shu (Livre des Liang), juan 50, p. 710-712, et le Nanshi (Histoire [des dynasties] du Sud), juan 72, p. 17811782 (mes références aux histoires dynastiques se rapportent toutes aux éditions de la Zhonghua shuju). Ayant « acquis une parfaite connaissance des canons et traités bouddhiques, [Liu Xie] s'employa à la classification de ces textes selon 
- au ton plat et non infléchi - wen (littérature/culture/ornement), xin (cœur/esprit/essence), diao (graver, ciseler/orner) et long (dragon) s'associent en effet selon une " vraie-fausse " symétrie, que l'on pourrait appeler plus simplement parallélisme formel. Loin de prétendre donner de manière satisfaisante la quadrature de ce cercle, mais parce que le titre emblématique de cet ouvrage fameux résiste si âprement aux tentatives de traduction et qu'il fut l'objet de tant d'interprétations différentes, je voudrais en proposer une sorte de long commentaire composé, afin de rendre hommage à la double et géniale invention que constitue l' "enseigne » du Wenxin diaolong. Il s'agira avant tout de discuter le sens et la valeur de chacune des composantes de ce titre dans une perspective historique, ainsi que l'ensemble de la définition qu'en donne Liu Xie lui-même dans les premières lignes de sa postface - «Xuzhi » (Déclaration d'intention) ${ }^{3}$-, mais aussi d'examiner les différentes traductions proposées par les savants modernes à la lumière de critères linguistiques, des usages anciens en matière de titres d'ouvrages, ou encore des usages particuliers observés chez les préfaciers et commen-tateurs du Wenxin diaolong lorsqu'ils citent son titre sous une

leurs différentes catégories, et en fit un catalogue pourvu de préfaces [...]. L'écrivain qu'était [Liu] Xie excellait surtout en matière de bouddhisme, aussi lui demandait-on toujours de composer les notices et les steles relatives aux temples, stûpa et moines illustres de la capitale. » Liu Xie fut le principal collaborateur laïc de l'éminent moine Sengyou (445-518), compilateur du Chu Sanzang ji ji (Collection des notices concernant la traduction du Tripitaka) et du Hongming ji (Recueil d'écrits proclamant la grandeur et la clarté [du bouddhisme]), entre autres œuvres bouddhiques.

3 Le cinquantième et dernier chapitre du Wenxin diaolong est l'un des plus abondamment traduits en langues occidentales. Outre les traductions complètes du traité par V. Yu-chung Shih (The Literary Mind and the Carving of Dragons. A Study of Thought and Pattern in Chinese Literature, Hong-Kong, The Chinese University Press, 1983), A.C. Lavagnino (Il tesoro delle lettere : un itaglio di draghi, Milan, Luni, 1995), A. Relinque Eleta (El corazón de la literatura y el cincelado de dragones, Grenade, Comares, 1995), et Wong Siu Kit, Lo Chung Hang, Lam Hwong Tai [The Book of Literary Design (Wenxin diaolong), Hong Kong, Hong Kong University Press, 1999], il faut citer celles de G. Yang et Yang Hsien-yi («Carving a Dragon at the Core of Literature », Chinese Literature, 8, 1962, p. 5871), et S. Owen («What I had in Mind : Afterword », Readings in Chinese Literary Thought, Cambridge (Mass.), Harvard University Press, 1992, p. 292-298). 
forme abrégée. Car la glose qui va suivre, assurément prolixe s'agissant de quatre seuls caractères, sera autant nourrie des problèmes d'interprétation qui se posent singulièrement à tout lecteur occidental que de ceux dont s'occupe toute exégèse dans la plus « ordinaire » tradition lettrée.

\section{L'esprit de littérature}

[Ce que je nomme] esprit de littérature est l'esprit qui s'applique à la création littéraire. Jadis, Juanzi [composa] un Esprit de la cithare ${ }^{4}$ et Wangsun l'Esprit d'ingéniosité 5 . Ah ! Que [le mot] esprit est excellent... C'est pourquoi j'en [veux] user ici. ${ }^{6}$

4 Qinxin. La biographie de Juanzi dans le Liexian zhuan (Biographies des immortels) de Liu Xiang (77?-6 av. J.-C.) mentionne son Esprit de la cithare en trois chapitres, "d'une composition harmonieuse », ou "qui contient des instructions musicales » (Wang Shumin, Liexian zhuan jiaojian, Taipei, Zhongyang yanjiuyuan, Zhongguo wenzhe yanjiusuo, 1995, p. 24 ; traduction de M. Kaltenmark, Le Lie-sien Tchouan [Biographies légendaires des Immortels taoïstes de l'antiquité], réimp. de l'éd. de 1953, Paris, De Boccard, 1987, p. 69 et 70, n. 6). Juanzi se manifesta dans le pays de Qi à l'âge de trois cents ans ; il aimait absorber de l'atractyle et savait commander au vent et à la pluie. Il composa également un Tianren jing (Livre du ciel et de l'homme) en quarante-huit chapitres. Le traité bibliographique du Han shu (Livre des Han, juan 30, p. 1730) recense parmi les leçons de maîtres de la tradition taoïste un Juanzi* en treize chapitres, dont l'auteur avait pour nom personnel Yuan, était originaire du royaume de Chu et disciple de Laozi. Au sujet des traditions relatives aux différents personnages nommés Juan, Juan* ou encore Huan, cf. M. Kaltenmark, op. cit., p. 70-71.

5 Qiaoxin. Le Han shu (juan 30, p. 1725) recense parmi les leçons de maîtres de la tradition des lettrés un "Wangsunzi en un chapitre, autrement appele Qiaoxin ». D'après le traité bibliographique du Sui shu (Livre des Sui, juan 34, p. 997), cet ouvrage existait encore sous les Liang, avant d'être perdu. Les fragments du Wangsunzi subsistant dans différentes encyclopédies des Tang (cf. Ma Guohan [1794-1857], Yuhan shanfang ji yishu, vol. 4, p. 2545-2546), consistent en anecdotes et propos exemplaires sur l' art de gouverner et la sagesse du souverain. On y voit intervenir Confucius et Zixia, mentionnés les tyrans Jie et Zhou, ainsi que cinq personnages apparaissant dans le Chunqiu et le Zuozhuan entre les années 612 et 476 av. J.-C.

6 Wenxin diaolong, «Xuzhi », L, p. 1898-1899 (toutes mes références au texte 
Dès sa première phrase, la postface du Wenxin diaolong entre dans le vif du sujet et définit le premier terme du titre du traité par une référence à l'incipit de la préface au « Wen fu » (Fu sur la composition littéraire) de Lu Ji (261-303) : «Chaque fois que je lis les œuvres des écrivains de talent, je crois pouvoir comprendre ce à quoi s'appliquait leur esprit (yongxin). » 7 Bien que Liu Xie ne se soit pas privé de porter ailleurs un jugement dépréciatif - assurément injuste, mais en un sens de bonne guerre - sur l'œuvre d'un illustre prédécesseur qu'il prétendait évidemment dépasser ${ }^{8}$, cette référence devait sembler fort naturelle aux lecteurs de sa « Déclaration d'intention », car le « Wen fu » était sans aucun doute le plus célèbre texte de théorie littéraire à cette époque. Le futur empereur Jianwen des Liang, Xiao Gang (503-551), userait à son tour de plusieurs allusions limpides au «Wen fu » dans sa «Lettre au prince de Xiangdong » : il établirait notamment la différence entre les poètes anciens et plus récents « en

du Wenxin diaolong mentionnent le titre du chapitre, son numéro en chiffres romains et la pagination de l'excellente édition annotée de Zhan Ying, Wenxin diaolong yizheng, Shanghai, Shanghai guji chubanshe, 1989, 3 vol.).

7 «Wen fu » inclus dans Xiao Tong (Zhaoming taizi, 501-531), Wenxuan (Anthologie, éd. de Hu Kejia (1757-1816), réd. Pékin, Zhonghua shuju, 1977), juan 17, p. 1b. Je reprends partiellement la traduction de Kôzen Hiroshi ( « Le Fu sur les lettres de Lu Ji » in J.-P. Drège [éd.], De Dunhuang au Japon. Études chinoises et bouddhiques offertes à Michel Soymié, Paris, Droz, 1996 p. 376) : «Chaque fois que je lis les ouvres des écrivains de talent, j' arrive à comprendre en mon cour leur manière de penser. »

8 Lorsque, dans le même chapitre, Liu Xie énumère les défauts et lacunes des œuvres de critique littéraire qui ont précédé le Wenxin diaolong pour mieux justifier la nécessité et la méthode de son traité, il qualifie le «Wen fu » d' « ingénieux mais désordonné " (« Xuzhi », L, p. 1918). Cf. également les chapitres «Zongshu » (De l'art littéraire en général, XLIV, p. 1631) et «Lunshui » (Dissertation et persuasion, XVIII, p. 719). Les accusations portées par Liu Xie sont évidemment injustes compte tenu de l'ambition, de la forme et de la réussite absolue de l'œuvre de $\mathbf{L a ~} \mathbf{J i}$, mais aussi d'une profonde communauté de vues théoriques sur la création littéraire entre l'auteur du Wenxin diaolong et son prédécesseur. 
Variations sur le titre du Wenxin diaolong
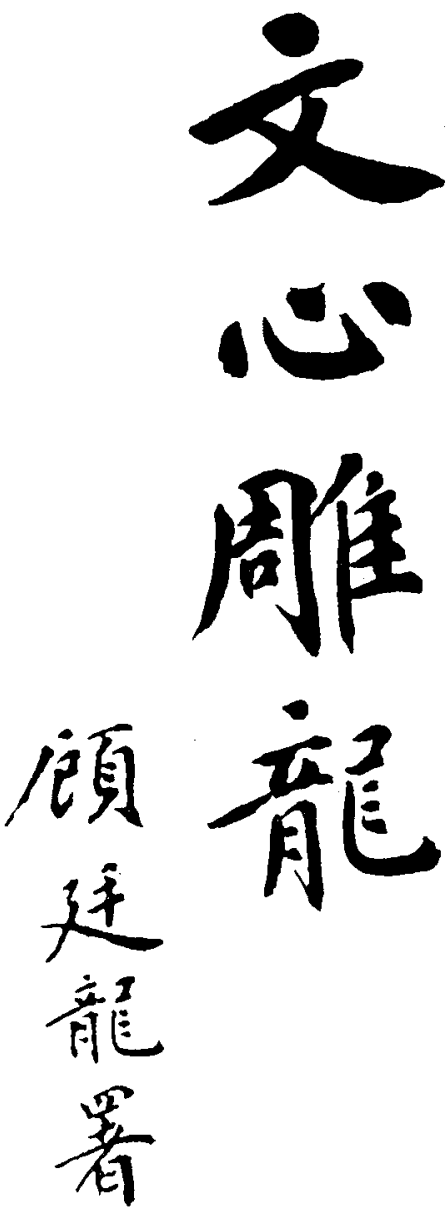

证过 
considérant leur façon de dispenser les expressions (yici) ${ }^{9}$ et ce à quoi s'appliquait leur esprit ». 10

Chez Lu Ji comme chez Liu Xie, l'expression yongxin peut être comprise de diverses manières : l'usage de l'esprit (ou cœur) peut être la pensée (le sentiment) qui l'occupe, l'intention qu'il conçoit, ou encore l'effort d'un exercice, d'une activité intellectuelle qui l'anime (et par suite la concentration, l'application). Ce dernier sens est celui que revêt l'expression yongxin dans un fameux propos de Confucius :

Regardez ces gens qui se gavent à longueur de journée, sans faire le moindre usage de leur esprit : c'est à en désespérer ! N'y a-t-il pas des jeux d'échecs ou de dames? Qu'ils y jouent, cela vaudrait encore mieux que rien. ${ }^{11}$

Cependant, lorsque Shun interrogeait Yao selon le Zhuangzi, il ne lui demandait pas grâce à quelle saine activité intellectuelle le Fils du Ciel faisait travailler ses méninges :

«Qu'en est-il des préoccupations (yongxin) du souverain céleste ? » Yao répondit : « Je ne dédaigne pas ceux qui n'ont pas le moyen de se plaindre,

9 L'emploi de cette expression complète la citation de la préface du "Wen fu », dont la deuxième phrase dit : " [Bien que la façon dont] ils disposaient les mots et dispensaient les expressions fût infiniment variée, il est possible d'en dire la beauté ou la laideur, les qualités et les défauts » (Wenxuan, juan 17, p. 1 b).

10 "Yu Xiangdong wang shu », Liang shu, juan 49, p. 690. Plus loin (p. 691), Xiao Gang emploie également l'expression qiaoxin (esprit ingénieux) qui se trouvait déjà dans le «Wen fu » (Wenxuan, juan 17, p. 8a). Contrairement à ce qu'indiquent les éditeurs modernes du Liang shu lorsqu'ils soulignent ces deux caractères comme un titre dans la " Lettre au prince de Xiangdong ", ni Lu Ji ni Xiao Gang ne semblent se référer directement à l'ouvrage de Wangsunzi (L'Esprit d'ingéniosité) auquel Liu Xie fait allusion pour expliquer l'emploi du mot xin dans le titre du Wenxin diaolong. Mais il est probable que Liu Xie se soit d'autant plus amusé à citer le Qiaoxin, sachant que tout lecteur du « Wen fu » pouvait entendre là encore résonner son écho.

11 Lunyu, 17.19 ; traduction d'A. Cheng (Entretiens de Confucius, Paris, Le Seuil, 1981), p. 139. 
et ne néglige pas le peuple indigent. Je souffre pour les morts, console leurs jeunes enfants et plains leurs veuves. Voilà tout ce qui me préoccupe. $»^{12}$

Manifestement Shun s'enquerait des sentiments et pensées où s'impliquaient le cœur et l'esprit de Yao - de ce à quoi il portait attention, de ce dont il se préoccupait en tant que souverain. La touchante compassion, l'amour de son peuple que révélait la réponse de Yao firent sans doute que, par la suite, on cita en exemple la « dévotion » de Yao et Shun en usant encore du terme yongxin ${ }^{13}$.

L'usage de l'esprit est l'objet d'une interrogation dans le Zhuangzi comme il est objet de connaissance dans le « Wen fu »: il n'est pas une pure activité qui vaudrait en tant qu'effort intellectuel ainsi que dans le Lunyu, mais une application à quelque chose (susceptible d'être défini ou compris) dans le processus d'une activité donnée - l'art de gouverner quant à Yao, celui d'écrire chez Lu Ji ${ }^{14}$. Lorsque Liu Xie invoque à son tour l'usage de l'esprit, il énonce explicitement l'activité qui en est le contexte, la chose à laquelle s'applique l'esprit : la création littéraire (weiwen zhi yongxin) - à l'instar de Lu Ji. C'est pourquoi il ne semble pas opportun de comprendre sa phrase en vertu d'un usage plus moderne de l'expression yongxin - même si cet usage est annoncé par le Lunyu - comme « effort ou travail intellectuel que représente la création littéraire », « application ou concentration de l'esprit que nécessite la création littéraire ».

12 Zhuangzi, «Tiandao », inclus dans Xinbian zhuzi jicheng, Pékin, Zhonghua shuju, 1989 , p. 475.

13 Cf. l'une des réponses de Dong Zhongshu (ca. 179-93 av. J.-C.) à l'examen de l'empereur Wu des Han (r. 141-87) (Han shu, juan 56, p. 2512), ou encore le « Jitian fu » (Fu sur le champ sacré, Wenxuan, juan 7, p. 15b) de Pan Yue (247300 ).

14 Le commentaire de Li Shan (?-689) à l'incipit du «Wen fu » cite bien le Zhuangzi et non le Lunyu. Dans un autre texte de Lu Ji («Diao Wei Wudi wen » [Lamentation pour l'empereur Wu de Wei], Wenxuan, juan 60, p. 18a), le fait de ne pas « appliquer son esprit à » quelque chose est mis en parallèle avec celui d'« en parler rarement». 
On peut en revanche hésiter à faire du composé weiwen une préposition suivie d'un nom, plutôt qu'un verbe accompagné de son objet : à comprendre l'« esprit qui s'applique à la littérature » plutôt que l' « esprit qui s'applique à la création littéraire $\gg{ }^{15}$. Quand Liu Xie dénombre les chapitres de son traité dans la «Déclaration d'intention», il précise que «quarante-neuf chapitres seulement s'occupent de littérature » en employant le composé weiwen comme un groupe prépositionnel (weiwen yong) ${ }^{16}$. Néanmoins, la structure de sa définition de l'esprit de littérature (weiwen zhi yongxin) est bien différente et l'emploi du composé weiwen pour désigner le fait de «composer une œuvre littéraire » extrêmement fréquent. On en trouve plusieurs exemples dans des textes déjà cités : la «Lettre au prince de Xiangdong " 17 où Xiao Gang se dit modestement « malhabile à la composition littéraire » (ou poétique ; zhuo yu weiwen), ou encore la biographie de Liu Xie dans le Liang shu: "L'écrivain qu'était [Liu] Xie excellait surtout en matière de bouddhisme » (Xie weiwen chang yu foli $)^{18}$, mais également dans d'autres textes tout aussi célèbres pour l'histoire de la critique littéraire. Dans sa «Lettre à Yang Dezu (Yang Xiu, 175-219) », Cao Zhi (192-232) ${ }^{19}$ avoue qu'il aima depuis sa plus tendre enfance composer des œuvres littéraires » (hao weiwenzhang), tandis que Huangfu

15 La première lecture est celle que suppose le préfacier du Wenxin diaolong, Ye Lianfang (préface datée de 1545 et reproduite par Wang Liqi, Wenxin diaolong xinshu, Pékin, Université de Paris, Centre d'études sinologiques de Pékin, 1952, p. 142), lorsqu'il paraphrase la définition de Liu Xie : « L'esprit de littérature, [cela signifie] appliquer son esprit à la littérature (yongxin yu wen). »

16 Et en prenant modèle sur le commentaire Xici du Yijing, cf. "Xuzhi », L, p. 1930.

17 « Yu Xiangdong wang shu », Liang shu, juan 49, p. 690.

18 Ou plus littéralement " [Liu] Xie, pour ce qui était de la composition littéraire, excellait surtout en matière de bouddhisme », Liang shu, juan 50, p. 712.

19 "Yu Yang Dezu shu ", Wenxuan, juan 42, p. 12a. Cependant, la plus ancienne version de cette lettre (celle du Dianliue, cité par le commentaire de Pei Songzhi [372-451] à la biographie de Cao Zhi dans le Sanguo zhi, Wei shu, juan 19, p. 558) ne comporte pas l'expression weiwenzhang : Cao Zhi depuis son enfance « aima les fu (hao cifu). » 
Mi (215-282) parle dans la « Préface aux Fu des trois capitales » de Zuo Si (ca 250-ca 305) des «écrivains du passé » (xi zhi weiwenzhe $)^{20}$.

De même que le « Wen fu » traite de l'art d'écrire plus que des belleslettres, Liu Xie définit l'esprit de littérature comme celui qui «s'applique à la création littéraire ». Cela étant, il ne s'est pas contenté de livrer au lecteur du Wenxin diaolong une explication univoque du premier terme de son titre; il y a ajouté une sorte de glose, ou variation sur le thème - à la gloire - du xin qu'il faut maintenant examiner. Lorsqu'il cite les Qinxin et Qiaoxin, leçons des maîtres Juanzi et Wangsunzi censément très anciennes, Liu Xie affecte de justifier le fait d'avoir voulu « user » du terme $x$ in dans le titre de son traité, en rappelant de quels titres de noblesse peut se prévaloir ce mot « excellent ». On remarque qu'il a ce faisant équitablement inséré son propre ouvrage dans la double tradition des écoles lettrée et taoïste, de même que les deux grandes parties du Wenxin diaolong commencent chacune sous les auspices révérencieuses de l'une et l'autre tradition ${ }^{21}$.

Mais si le wenxin est "l'esprit qui s'applique à la création littéraire ", le lecteur est d'abord censé comprendre que le Qinxin traitait de l'esprit qui s'applique à l'art de la cithare, et le Qiaoxin de celui qui s'applique à être ingénieux. Pourtant, découvrant dans la phrase suivante que Liu Xie

20 « 'Sandu fu' xu », Wenxuan, juan 45, p. 28b. On peut encore citer, en prélude à un prochain développement, le « Wei Fan shangshu rang libu fenghou diyi biao » (Premier mémoire écrit à la demande du [membre de] la chancellerie Fan [Yun] pour refuser [sa nomination au département de] la fonction publique et son inféodation comme marquis, ibid., juan 38, p. 15b) de Ren Fang (460508), qui raconte avec modestie la formation litteraire de Fan Yun (451-503): en vain il « $s$ 'exerçait à la composition " comme un enfant au style sigillaire (zhuanke weiwen).

21 Les trois premiers chapitres posent les Classiques comme origine de toute littérature et comme critère absolu de toute critique littéraire, tandis que le vingt-sixième ( Shensi ») - inaugurant la seconde partie du traité — définit cette notion fondamentale de la théorie littéraire de Liu Xie qu'est l'inspiration ou pensée créatrice en se référant résolument au Zhuangzi (cf. infra, n. 33). Dans le même sens encore, la « Déclaration d'intention » est toute empreinte de pensée dite confucéenne, mais se conclut par une clausule rimée marquant l'allégeance de l'auteur au Zhuangzi. 
énonce pour la cinquième fois ce terme désormais emblématique, et s'exclame avec emphase et exaltation devant l'excellence du mot xin, si beau et magique que l'auteur ne put faire autrement qu'en user (yong, s'y «appliquer » $)^{22}$, le même lecteur entend clairement l'intention qu'on veut lui signifier ainsi : on joue sur les mots, et celui de xin est si excellent qu'on peut l'employer dans toute sa richesse. Il est donc naturellement porté à lire les premières phrases de la « Déclaration d'intention» comme une variation sur divers sens du mot xin, et autorisé à confronter la définition initiale d'un esprit appliqué à quelque chose, avec d'autres valeurs sémantiques attachées au « cœur » : en l'occurrence l'origine, le fondement, la quintessence. Car les composés qinxin, qiaoxin et wenxin peuvent assurément se comprendre et se traduire comme l' « essence » - l'art en termes occidentaux - de la cithare, de l'ingéniosité, de la littérature.

Sachant la vogue et la diffusion du vocabulaire et de la pensée bouddhiques à l'époque de Liu Xie, ainsi que la familiarité de ce dernier avec l'un comme avec l'autre, on ne s'arrêtera pas en si bon chemin : l'excellence du mot xin ne tient-elle pas également à son importance dans le bouddhisme, comme notion suprême recouvrant l'ensemble des phénomènes spirituels, $s$ 'opposant aux apparences ( $s e$ ) et gouvernant les dix mille lois des trois mondes? Plusieurs savants modernes ont même formulé et discuté la supposition suivante : c'est sous l'influence de l'Abhidharma-hrdaya-śâstra (Apitan xin lun ou Wubifa xin lun, Traité sur le fondement de la loi sans pareille) du moine indien de l'école des Sarvâstivâdin Dharmajina (d.i.), traduit par Sanghadeva (IV siècle) et Huiyuan (334-416) en 391 au mont Lu, que Liu Xie aurait usé du mot xin dans le titre de son propre traité. Fan Wenlan a le premier énoncé cette

22 On doit assurément entendre un jeu de mots entre les deux occurrences du verbe yong dans les premières lignes de la «Déclaration d'intention » en vertu duquel on pourrait traduire : « [Ce que je nomme] esprit de litterature est l'esprit dont on fait usage dans la création littéraire [...]. Ah ! Que l'esprit est excellent... C'est pourquoi j' ai usé de [ce mot] ... et fait ici usage de mon esprit. » Dans le contexte d'une explication du titre du Wenxin diaolong, le sens de la dernière phrase doit cependant être univoque, et ma traduction privilégie donc la lecture «terminologique ». 
hypothèse dans son commentaire de la « Déclaration d'intention » ${ }^{23}$, en y ajoutant un rapprochement entre la structure du Wenxin diaolong et celle de l'Abhidharma-hrdaya-sâstra, entre les clausules rimées (zan) du premier et les ghatta bouddhiques du second. Kôzen Hiroshi ${ }^{24}$ a réfuté cette comparaison formelle, mais, tout en soulignant la divergence des explications de Liu Xie et Huiyuan (dans sa préface à l'Abhidharma-hrdayaśâstra ${ }^{25}$ quant à la présence du mot xin dans le titre des deux traités, accrédite l'hypothèse de Fan Wenlan en ce qui concerne le titre du Wenxin diaolong, dans la mesure où il considère que son premier chapitre ( « Yuandao » [Le dao pour origine], voir ci-dessous) traite de l'origine de la littérature (wen, beauté ornementale, culture) en attribuant au xin le rôle de " fondement $" 26$.

Si l'on peut donc à la fois entendre les sens d'esprit, d'essence et de fondement dans le mot xin, le traducteur de la «Déclaration d'intention » se trouve confronté à un problème sans solution. Ou bien il contourne la difficulté en proposant plusieurs sens pour chaque occurrence du terme potentiellement polysémique, voire se contente d'une transcription non traduite, et laisse béante la question de la définition du composé wenxin.

23 Cf. Fan Wenlan, Wenxin diaolong zhu, 1936, réimp. Taipei, Taiwan kaiming shudian, 1958, juan 10, p. 22a, note 2.

24 «Bunshin chôryû to Shussanzô kishâu »(Le Wenxin diaolong et le Chu Sanzang ji ji), Chûgoku chûsei no shûkyô to bunka, Kyôto, Kyôto daigaku jinbun kagaku kenkyujô, 1982, p. 134-136.

25 Selon Huiyuan : «Parce qu'il s'occupe universellement de l'ensemble des sûtra et recense leur immense assemblé, l'auteur appela [cet ouvrage] du nom de fondement (xin). » («Apitan xin xu » [Préface à l'Abhidharma-hrdaya]; Chu Sanzang ji ji, T. 2145, vol. 55, juan 10, p. 72c).

26 Dans un article postérieur ( "Wenxin yu Apitan xin », in Rao Pengzi [dir.], Wenxin diaolong yanjiu huicui, Shanghai, Shanghai shudian, 1992, p. 339-44), Rao Zongyi réfute également le rapprochement structurel proposé par Fan Wenlan, mais démontre la célébrité de l'Abhidharma-hrdaya-sâstra à l'époque de Liu Xie, particulièrement au monastère Dinglin - où l'auteur du Wenxin diaolong travailla et sans doute résida durant de longues années - et parmi les compilateurs du Chu Sanzang ji ji. Il conclut à l'identité d'emploi du mot xin dans le sens de "principes fondamentaux »-dans le titre des deux traités. 


\section{Valérie Lavoix}

Ou bien, il use de termes différents dans chaque mouvement de la variation sur le terme xin, au risque de rendre l'ensemble incompréhensible :

[Ce que je nomme] wenxin est l'esprit qui s'applique à la création littéraire. Jadis, Juanzi ${ }^{a}$ [composa] l'Essence de la cithare et Wangsun l'Essence de l'ingéniosité. Ah! Que [le mot] fondement est excellent... C'est pourquoi j'en [veux] user ici.

Enfin, il peut choisir une traduction univoque, qui fausse légèrement mais équitablement le sens de chaque mouvement, et a le mérite de préserver la sensation du jeu de mots :

[Ce que je nomme] cœur de la littérature est le cœur qui s'applique à la création littéraire. Jadis, Juanzi [composa] le Coeur de la cithare, et Wangsun le Couur de l'ingéniosité. Ah! Que [le mot] cour est excellent... C'est pourquoi j'en [veux] user ici.

La solution que j'ai adoptée est nécessairement imparfaite. Elle suppose que la valeur bouddhique du terme xin et l'Abhidharma-hrdaya-śâstra ne jouent pas ici le rôle d'une référence cachée mais tout au plus celui d'une résonance, d'un écho secondaire ou troisième. D'autre part, elle consiste à choisir un sens univoque - même s'il ne convient pas idéalement dans le cas des leçons de maîtres Qinxin et Qiaoxin - considéré comme primordial eu égard au titre et au contenu du Wenxin diaolong. Car, dans la mesure où ce titre est un programme, il faut bien prendre en considération la valeur et le rôle que l'ensemble du traité attribue au mot xin. Or, dans l'immense majorité de ses occurrences, et plus précisément dans les passages ou chapitres cruciaux du traité, Liu Xie emploie ce terme sans outrepasser le champ sémantique qu'il recouvre classiquement en chinois comme « cour/ esprit ", siège indistinct du sentiment, de la volonté et de l'intellect, de la pensée et de la conscience ${ }^{27}$.

27 Dans une étude sur le vocabulaire technique du Wenxin diaolong (Wenxin diaolong wenlun shuyu xilun, Taipei, Huazheng shuju, 1981, p. 112-128), Wang Jinling a par exemple classé soixante-quatorze occurrences du mot xin sous les significations « idée », «pensée », « aspiration » et «cœur », et seulement quatre autres sous celles de « essence », « origine », « sève ». 
Si l'on veut bien survoler l'ensemble du Wenxin diaolong depuis son chapitre initial jusqu'à la clausule rimée de la " Déclaration d'intention », en passant par le crucial chapitre XXVI, «Shensi » (Inspiration), on aura un aperçu de la manière dont le traité joue des valeurs du mot xin, et de son sens «spirituel». Dans «Le dao pour origine » ${ }^{28}$, Liu Xie expose l'avènement originel de la littérature selon la voie de la nature - depuis l'ornement (la beauté, wen) du dao que sont les couleurs et les formes, les astres dans le ciel, les monts et rivières sur la terre, jusqu'à l'ornement (wen) des hommes qu'est la littérature - ; dans le même temps, il installe la notion d'esprit au cceur de l'univers et comme agent humain de la création littéraire :

[L'homme] est en vérité le cœur (noyau) du ciel et de la terre ${ }^{29}$. Dès lors que ce cœur (esprit) prit naissance, la parole fut instituée. Dès lors que la parole fut instituée, la littérature resplendit. ${ }^{30}$

Dragons et phénix, tigres et léopards, les nuages comme les plantes, le vent dans la forêt et les pierres roulant dans le ruisseau manifestent tous des beautés ornementales (wen) que l'art ne parvient pas à égaler :

Puisque même les choses dénuées de conscience arborent une telle profusion d'embellissements, comment l'être pourvu d'un esprit (xin) pourrait-il être privé d'ornement (wen, littérature) ? ${ }^{31}$

Est-il nécessaire enfin de préciser - par la grâce d'un jeu de mots limpide - que si seuls les hexagrammes Qian et Kun (ciel et terre) du Yijing furent dotés d'un commentaire intitulé Wenyan (Paroles ornées), cela prouve bien que :

28 «Yuandao ». Cf. la traduction et l'étude de ce chapitre par F. Jullien dans La Valeur allusive. Des catégories originales de l'interprétation poétique dans la tradition chinoise (Contribution à une réflexion sur l'altérité interculturelle), Paris, École Française d'Extrême-Orient, 1985, p. 35-56.

29 Citation du Liji (Mémoire sur les rites), «Liyun », inclus dans Shisanjing zhushu, Pékin, rééd. Zhonghua shuju, 1980, p. 1424b).

30 «Yuandao », I, p. 4.

31 lbid., p. 10. 


\section{Valérie Lavoix}

L'ornementation de la parole (yan zhi wen) est l'esprit (xin) même du ciel et de la terre. ${ }^{32}$

Au cœur du Wenxin diaolong, Liu Xie a placé - et déplacé hors de son contexte - cette citation du Zhuangzi :

Mon corps demeure au-dessus des fleuves et de la mer, mais mon esprit (xin) réside au pied des tours [du palais] de Wei. ${ }^{33}$

Elle introduit le chapitre «Shensi » et sert de définition à cette pensée créatrice, ou pouvoir de réflexion (conception, si) de l'esprit créateur (shen) qui lui permet de transcender les limites du temps et de l'espace et de «voyager avec les choses de l'univers » ${ }^{34}$. Au fil du développement, le sens du mot xin oscille entre les deux pôles du shen et du si : il désigne l'esprit à l'œuvre dans la création littéraire - dont la puissance (xinli), par exemple, peut s'aider d'une vaste expérience associée à la faculté de concentration, remèdes à la pauvreté du contenu et à la confusion ${ }^{35}$-, mais aussi la pensée qui est le fruit de cette mise en œuvre, celle notamment qui se dérobe (dunxin) lorsque le verrou de l'esprit créateur est fermé ${ }^{36}$.

Les premiers vers de la clausule résument finalement le moteur et la manière de l'inspiration, en établissant la primauté du shen sur le xin:

Quand l'âme ${ }^{37}$ avec les phénomènes communique, du sentiment est engendrée la variation ;

32 Ibid., p. 11.

33 «Shensi », XXVI, p. 975. Cf. Zhuangzi, « Rangwang », p. 979, où cette parole est la plainte de Mou, fils du duc de Wei qui l'avait inféodé bien loin de son palais.

34 «Shensi », XXVI, p. 976. Au sujet de ce chapitre, cf. l'article de F. Jullien, "Naissance de l'"imagination", Essai de problématique au travers de la réflexion littéraire de la Chine et de l'Occident », Extrême-Orient ExtrêmeOccident, 7, 1985, p. 23-81, particulièrement p. 33-42.

35 Cf. « Shensi », XXVI, p. 1000.

36 Cf. ibid., p. 976.

37 En dépit de ses connotations chrétiennes, j'emploie ce terme pour traduire ici 
les choses par leur apparence sollicitent, l'esprit répond [alors] au fil de sa raison. ${ }^{38}$

Au terme du processus, lorsque la composition littéraire est achevée, l'esprit de l'écrivain a trouvé le lieu où s'appliquer et se confier, puisque l'cuvre est un "véhicule» capable de transporter la pensée. Liu Xie peut ainsi conclure l'ensemble de son traité par les derniers vers de la clausule de la «Déclaration d'intention » :

Si littérature sait porter la pensée (xin), mon esprit (xin) [ici même] trouve où se confier. ${ }^{39}$

Il n'est pas question d'essence, d'origine ou de fondement dans ces passages que Liu Xie a lui-même mis en valeur par la place qu'il leur a conférée. Nombreux sont les préfaciers et lecteurs du Wenxin diaolong qui prirent acte du rôle prédominant accordé à l'esprit par ce traité : «Sa doctrine fondamentale est l'esprit, c'est-à-dire l'exercice de la pensée au sein de l'âme. » ${ }^{40}$ Zhang Xuecheng (1738-1801) soulignait également :

Les auteurs anciens qui ont traité de la littérature dissertaient exclusivement de l'expression littéraire (wenci). Monsieur Liu Xie fit exception, car en s'inspirant des idées de Monsieur Lu Ji, il disserta excellemment de l'esprit de littérature (wenxin). ${ }^{41}$

Et bien qu'il soit très tentant de considérer le Wenxin diaolong comme un «Art littéraire », ou traité sur «L'essence de la littérature », mieux vaut se

shen, par commodité et bien que la «mobilité » de l'anima puisse sembler encore bien limitée comparée aux capacités infinies du shen - qui envahit les versants lorsqu'il gravit la montagne et inonde l'étendue des flots lorsqu'il contemple la mer.

38 «Shensi », XXVI, p. 1007.

39 «Xuzhi », L, p. 1938.

40 Préface au Wenxin diaolong de Cao Xuequan (1574-1647), datée de 1612, et reproduite par Wang Liqi, Wenxin diaolong xinshu, p. 148.

41 Wenshi tongyi (Principes généraux de la littérature et de l'histoire), « Neipian », juan 3, « Wende », inclus dans Ye Ying, Wenshi tongyi jiaozhu, Pékin, Zhonghua shuju, 1985, p. 278. 


\section{Valérie Lavoix}

conformer à l'avis des auteurs passés comme à la majorité des traducteurs occidentaux du titre du traité (j'y reviendrai) pour comprendre que le Wenxin disserte sur l' « esprit de littérature ».

Le terme français « esprit » reste cependant un équivalent très imparfait eu égard aux divers sens du mot xin dans la langue chinoise en général, et dans les premières lignes de la « Déclaration d'intention » en particulier. Il recouvre par ailleurs dans la tradition occidentale des emplois et des significations forcément inappropriés ici. Pourtant, il a également l'avantage d'en concilier plusieurs autres qui conviennent assez bien dans le cas d'un esprit appliqué à la création littéraire, transporté par la littérature ou qui trouve à se confier dans l'œuvre littéraire. De «l'âme comme agent des pensées, souvenirs, volontés », "il se dit en particulier des facultés intellectuelles, de l'aptitude à comprendre, à saisir, à juger ", mais aussi des "principes, motifs, impulsions, tendances, d'après lesquelles on se dirige » ${ }^{42}$. Par ailleurs, l'esprit peut être une « aptitude, disposition (l'esprit des affaires, l'esprit mathématique) », ou encore le « sens d'un auteur, d'un texte, etc. [...], le caractère d'un auteur ». Enfin, puisqu'il s'agit ici de l'esprit de littérature ${ }^{43}$, deux expressions (très) françaises font entendre une résonance qui peut sembler opportune. Ce sont tout d'abord les « ouvrage de l'esprit, ouvrage d'esprit : tout ce que les hommes inventent dans les sciences et dans les arts est un ouvrage de l'esprit. Les compositions des gens de lettres sont des ouvrages d'esprit. » Et parce que le wen est aussi la beauté, l'ornement, le raffinement et la culture, on ne résiste pas au plaisir de solliciter le « bel esprit, la culture des belles-lettres, de la littérature »ce bel esprit dont la «carrière épineuse » suscitait chez Boileau (16361711) la mise en garde que Liu Xie n'aurait peut-être pas reniée :

42 Ces définitions, ainsi que les suivantes, sont extraites du Littré («Esprit»).

43 S'il existe un esprit mathématique, on pourrait se contenter de parler d'esprit littéraire. En employant ici la particule ambivalente « de », je cherche à éviter une compréhension trop univoque ou réductrice du composé wenxin : à rendre compte du sens actif, dynamique et potentiellement multiple que lui confèrent à la fois sa définition comme «esprit appliqué à la création littéraire » et les divers emplois conjoints des termes wen et xin dans le Wenxin diaolong, dont j'ai cité plus haut quelques exemples. 


\title{
Variations sur le titre du Wenxin diaolong
}

\begin{abstract}
N'allez pas sur des vers sans fruit vous consumer,
$\mathrm{Ni}$ prendre pour génie un amour de rimer :

Craignez d'un vain plaisir les trompeuses amorces,

Et consultez longtemps votre esprit et vos forces. ${ }^{44}$
\end{abstract}

\section{Dragons et vermisseaux}

Depuis toujours, des ouvres de littérature, la forme est composée comme on cisèle un ornement ; prendrai-je pour modèle le dragon ciselé qui faisait de Zou Shi ${ }^{45}$ la réputation ? ${ }^{46}$

44 Art poétique, Chant I, Boileau: Euvres 2, Paris, Garnier Flammarion, 1969, p. 87.

45 Sous les Royaumes combattants, Zou Shi était l'un des savants lettrés qui s'assemblaient au pied de la porte Ji (ou dans l'académie Jixia) à la capitale du royaume de Qi et « composèrent des ouvrages traitant de l'art d'établir la paix » (Shiji [Mémoires historiques], juan 74, p. 2346). Le traité bibliographique du Han shu (juan 30, p. 1733) recense un Zoushizi en douze chapitres, dans la tradition yinyang, attribué à un « homme de Qi surnommé Shi le graveur de dragons. » Dans le Wenxin diaolong, le chapitre « Shixu » (Le cours de l'histoire, $\mathrm{XLV}$, p. 1662) rappelle également que «Zou Shi connut la renommée pour ses dragons ciselés. »

46 «Xuzhi », L, p. 1899. La plupart des tratucteurs et commentateurs modernes du Wenxin diaolong comprennent la dernière proposition comme une question de rhétorique équivalant à une dénégation : « Serait-il possible que je me réfère au dragon ciselé qui faisait de Zou Shi la réputation ? » devant signifier « Je ne veux évidemment pas m'y référer (seulement) ». Li Qingjia (Wenxin shiyu ji, Shanghai, Shanghai guji chubanshe, 1989, p. 95-6) remarque que cette dénégation, et la valeur dépréciative qu'elle confère à l'expression « dragon ciselé », paraissent peu logiques dans la mesure où elles succèdent à une proposition où l'ornement ciselé possède une valeur résolument positive - et ne sont elles-mêmes suivies d'aucune autre sorte d'explication. Que le second composé du titre du Wenxin diaolong soit expliqué par Liu Xie en un paradoxe si lapidaire et déceptif me paraît également curieux. Au lieu d'introduire une dénégation sous la forme d'une question de rhétorique, la particule interrogative $q i$ (est-il possible que, est-ce que, comment) peut aussi bien marquer une affirmation déguisée sous la forme d'une prétérition ( « feindre de ne pas vouloir faire ce que l'on fait néanmoins », selon B. Dupriez, Gradus, les procédés littéraires, Paris, 10/18, 1984, p. 360), proche en l'occurrence de la dubitation 


\section{Valérie Lavoix}

La « Déclaration d'intention » du Wenxin diaolong est assurément moins bavarde au sujet du second composé du titre du traité qu'au sujet du premier : Liu Xie se contente de poser un constat - les écrits littéraires sont composés comme on cisèle un ornement -, et d'évoquer l'emploi de l'image du «dragon ciselé » — ou « graveur de dragons » - à la fin des Royaumes combattants. Le constat n'a pas besoin d'être démontré : sa légitimité tient à l'évidence des métaphores artisanales (essentiellement attachées aux techniques de la gravure, de la fonte, du tissage et de la broderie) dans la langue et la pensée chinoises anciennes, qu'elles soient utilisées pour parler de la morale et de l'éducation personnelle ${ }^{47}$ ou de la culture et de l'accomplissement que manifestent les œuvres des hommes ${ }^{48}$. Parmi toutes les «façons » artisanales, celle qui consiste à manier le ciseau convient assurément à désigner par analogie l'art littéraire. Car l'ornement (wen) qui donne son nom à la littérature peut être un assemblage de couleurs ou de lignes, les veines du bois ou de la pierre, une marque sur le corps humain, un décor incisé sur le jade : il est le fruit d'un raffinement (relativement à l'essence, la simplicité, le naturel zhi), d'un tracé, d'une entaille. Quant à la gravure du jade (diao), elle désigne par extension le fait d'ajouter un ornement figuratif, ou l'embellissement qui en résulte - et qui peut être, tout comme le wen, jugé avantageux ou excessif selon les cas. Mais si l'on

(à l'instar de cet exemple cité par B. Dupriez d'après Valéry : «Oserai-je parler de la Chronologie [...] »). Que le titre du Wenxin diaolong fasse référence au plus ancien emploi connu de l'expression diaolong est une évidence que l'auteur avait à peine besoin de rappeler, et qu'il habilla donc par cette élégante tournure de rhétorique. Je tenterai par ailleurs de démontrer que le composé diaolong avait à l'époque de Liu Xie un sens suffisamment laudatif, sans ambiguiité, pour qu'il n'ait nul besoin de se défendre de quoi que ce soit.

47 Cf. par exemple le poème 55 du Shijing («Weifeng », «Qi ao », inclus dans Shisanjing zhushu, p. 321a), le Erya («Shixun », ibid., p. 2591b), le « Daxue » (Liji, ibid., p. 1673b) et le Lunyu, (1.15).

48 Le chapitre XXXII du Wenxin diaolong, le premier des chapitres traitant de manière purement technique de la composition littéraire et de la rhétorique, est une belle illustration de cet usage, puisqu'il est intitulé « Rongcai » (La fonte et la taille). 
en croit Yang Xiong (53 av. J.-C.-18 ap. J.-C.) : « Un jade parfait qui n'est pas gravé (diao) est comme un discours excellent dépourvu d'ornement (wen). »49

L'inventaire complet du lexique artisanal, et en particulier de celui de la gravure, dans les textes de la critique littéraire médiévale permettrait sans doute d'évaluer avec précision l'importance relative de ce lexique dans le Wenxin diaolong : il démontrerait que l'ensemble du traité illustre amplement l'emblème de son titre, mais qu'en termes de proportions, compte tenu de la longueur de l'ouvrage, l'importance de ce lexique n'est peut-être pas exceptionnelle. L'exception ou la nouveauté relative qui m'intéresse ici est plutôt celle du dragon ciselé : car s'il est un stéréotype que Liu Xie a su employer de manière originale, c'est bien celui-là. À la différence du composé wenxin, c'est moins le sens que la valeur de l'expression diaolong qui pose le plus de problèmes aux lecteurs et traducteurs du Wenxin diaolong, et c'est pourquoi il me semble utile de retracer l'histoire de cette expression et de ses emplois. Une semblable étude ne pourrait d'ailleurs pas être menée en ce qui concerne l' " esprit de littérature », puisqu'on n'en connaît tout simplement aucun usage avant Liu Xie : son invention est de ce point de vue absolue.

De la " gravure de dragons ", les plus anciennes occurrences connues sont déjà métaphoriques. Ainsi que le rappelle Liu Xie, cette expression fut tout d'abord un surnom ${ }^{50}$ : la réputation ou « étiquette » qui distingua parmi tous les auteurs de leçons de maîtres (zhuzi) le seul dont on vantât l'œuvre pour sa beauté littéraire et non pour sa pensée. Le graveur de dragons Zou Shi aurait été un talentueux écrivain, et tout d'abord un habile récupérateur

49 Fayan (Discours sur les normes), « Guajian » inclus dans Zhuzi jicheng, vol. 7, p. 19. Liu Xie ne manque pas de faire allusion à cette belle maxime dès le troisième chapitre du Wenxin diaolong («Zongjing», Les classiques pour modèles, p. 84), en rappelant que Yang Xiong définissait ainsi la nature ornée (littéraire) des cinq classiques.

50 À l'instar de Liu Xie, les commentateurs des textes où l'image de la gravure des dragons est employée se réferent toujours exclusivement à «Zou Shi le graveur de dragons » tel qu'il est nommé dans le Shiji : on ne connaît donc pas d'emploi antérieur de cette image. 


\section{Valérie Lavoix}

des idées de son contemporain Zou Yan (ca 305-240) ${ }^{51}$, également maître de la tradition yinyang, et qu'on appelait le «spécialiste (ou discoureur) du ciel ». On lit dans les biographies de Mengzi et Xunzi, selon le Shiji :

Zou Shi était l'un des maîtres Zou de Qi ; il recueillit un certain nombre des méthodes de Zou Yan pour les consigner par écrit. ${ }^{52}$

Les méthodes de Zou Yan consistaient à disserter avec ampleur [des sujets] les plus vastes et lointains ${ }^{53}$. Quant à Zou Shi, ses écrits étaient si parfaits qu'ils étaient difficiles à mettre en pratique. Chunyu Kun ${ }^{54}$ résida longtemps avec eux, et donnait souvent à entendre des discours excellents. C'est pourquoi les gens de Qi vantaient [leur talent] en ces termes : "Un spécialiste du ciel : [Zou] Yan; un graveur de dragons : [Zou] Shi ; une corne d'abondance : Chunyu Kun. » 55

Parce qu'elle était « difficile à mettre en pratique » (ou à enseigner), l'œuvre de Zou Shi acquit une réputation d'inefficacité et de futilité assurément

51 Le traité bibliographique du Han shu (juan 30, p. 1733) attribue à Zou Yan le " spécialiste du ciel » un Zouzi en quarante-neuf chapitres, et mentionne un Zouzi zhongshi (Achèvement et avènement de Zouzi) en cinquante-six chapitres qui appartiendrait à la même école.

52 Shiji, juan 74, p. 2347.

53 On peut également comprendre que ces méthodes «étaient impraticables à force de disserter avec [trop] d'ampleur ». Cette lecture s'associe assez bien avec la phrase suivante concernant Zou Shi. Le Shiji (juan, p. 2344) donne un aperçu des dissertations de Zou Yan, consistant à décrire l'univers dans ses dimensions illimitées, en partant du plus petit pour atteindre le plus grand, l'infini et l'inconnu, et précise que si ces démonstrations produisaient un grand effet sur les princes et dignitaires qui les entendaient, on ne fut plus capable d'appliquer ces méthodes par la suite.

54 Chunyu Kun était un autre savant de l'académie Jixia à Qi, très érudit sans pour autant avoir de spécialité. C'était avant tout un homme à la langue aussi habile que bien pendue, ce qui lui vaut d'apparaître non seulement avec Zou Yan et Zou Shi dans les biographies de Mengzi et Xunzi (Shiji, juan 74, p. 23462348), mais également parmi les beaux parleurs ou bouffons du Shiji (cf. « Guji liezhuan ", juan 126, p. 3209-3210).

55 Shiji, juan 74, p. 2348. Pour la louange des gens de Qi, je reprends la traduction de J.-P. Diény, Le symbolisme du dragon dans la Chine antique, p. 198. 
liée à la valeur péjorative que porte tout ornement (wen ou diao) lorsqu'il est considéré comme excessif - cette mauvaise réputation justifiant l'interprétation négative (culpabilisée ou culpabilisante ?) généralement proposée pour la glose du composé diaolong dans la "Déclaration d'intention " ${ }^{56}$. Sima Qian savait certes être caustique, mais on peut également souligner qu'à la différence des autres penseurs des Royaumes combattants, conseillers techniques et politiques des souverains qu'ils servaient, Zou Shi aurait été un écrivain avant tout et pourquoi pas le premier « maître littérateur » individuel de l'histoire - comme on dit de Qu Yuan qu'il en fut le premier poète «individuel ». À cet égard il allait sans nul doute à rebours des pratiques et - surtout - des besoins de son temps.

Que Sima Qian eût ou non une mauvaise opinion de Zou Yan ${ }^{57}$ et de Zou Shi, les gens de Qi ne semblent pas avoir été ironiques en faisant leur éloge. De même, les fragments du catalogue de Liu Xiang (77?-6) qui ont été conservés à leur sujet expliquent leur surnom de manière résolument laudative :

Zou Yan parlait de l'achèvement et de l'avènement des cinq vertus [des cinq agents] et de l'immensité de l'univers ; comme il traitait de tout ce qui concernait le ciel, on l'appelait le spécialiste du ciel. Zou Shi améliorait les écrits de [Zou] Yan; il les embellissait comme on grave et cisèle un décor de dragon, et c'est pourquoi on l'appelait le graveur de dragons. ${ }^{58}$

Liu Xiang dépliait ainsi la métaphore en la paraphrasant d'une comparaison, et rendait à l'expression diaolong son sens premier : la gravure d'un décor de dragon (longwen). L'art littéraire de Zou Shi était aussi minutieux et accompli que celui du graveur ; son œuvre alliait à la beauté même de l'art celle, suprême, du motif. Le dragon aux transformations et pouvoirs surnaturels échappant à toute règle, signe faste du mandat du ciel reçu par

56 Cf. supra, note 46.

57 Cf. supra, note 53.

58 «Bielu » (Registres particuliers), cité par le commentaire de Pei Yin ( $\left.v^{\mathrm{e}} \mathrm{s}.\right)$ au Shiji, juan 74, p. 2348. 
les anciens rois, ou de la vertu du souverain ${ }^{59}$, forme le décor privilégié des attributs (attelages) de l'empereur. Mais surtout, paré des cinq couleurs, d'un aspect plein de lumière et de magnificence, le dragon qui sert de décor est emblème de beauté redoublée dans la mesure où son corps porte luimême un beau décor (wenzhang) ${ }^{60}$. Ainsi le décor de dragon est-il une métaphore pour vanter le style de Cao Pi (187-226), futur empereur Wendi de Wei, sous la plume de Wu Zhi (177-230) : «Lorsque pour étaler les grâces [de son style] il abaisse le pinceau, c'est un décor de phénix et de dragons qui se déploie. " ${ }^{61}$ On ne s'étonne pas de voir le phénix s'allier ici au dragon ; ailleurs, quand la beauté d'une œuvre n'est pas comparée à celle d'un tigre peint ${ }^{62}$, c'est le serpent magique qui rivalise d'importance avec son cousin dans le bestiaire des métaphores classiques de la réussite littéraire ${ }^{63}$.

Le dragon ciselé resurgit quant à lui pour la première fois sous la plume de Cai Yong (132-192), peu avant Wu Zhi (en 184 très exactement) : ce qui semble être la plus ancienne occurrence de cette métaphore après le Shiji

59 Cf. les textes cités par J.-P. Diény, Le symbolisme du dragon dans la Chine antique, p. 26 et $72-73$ entre autres.

60 Cf. ibid., p. 26, 148-149, 198 notamment.

61 « Da Wei taizi jian » (En réponse au prince héritier de Wei), Wenxuan, juan 40, p. 19a (traduction de J.-P. Diény, Le symbolisme du dragon dans la Chine antique, p. 199).

62 Le frère de Cao Pi, Cao Zhi écrivit une lettre fameuse à Yang Xiu (175-219), où il se moqua vertement des vantardises poétiques de l'un des sept maîtres de Jian'an (Jian'an qizi), Chen Lin (?-217) : «[Chen Lin] s'adonne sans relâche au genre de l'exposition et s'est à maintes reprises déclaré capable de la même veine que Sima Zhangqing (Sima Xiangru, 179-117 av. J.-C.) - tel celui qui [prétendrait] peindre un tigre mais ne réussirait qu'à dessiner un chien " ("Yu Yang Dezu shu », Wenxuan, juan 42, p. 13a).

63 La perle donnée au marquis de Sui par le serpent qu'il avait soigné (cf. Gan Bao [actif 317-322], Soushen ji 453, juan 20, Pékin, Zhonghua shuju, 1979, p. 238, et R. Mathieu [dir.], À la recherche des esprits, Paris, Gallimard, 1992, p. 204) est citée par Cao Zhi dès le début de sa lettre à Yang Xiu : «En cette époque qui est la nôtre, chacun prétend tenir en main la perle du serpent magique ; tous déclarent posséder le jade du mont Jing » - trouvé par le sire He («Yu Yang Dezu shu », Wenxuan, juan 42, p. 12b). 
revêtait alors un sens plus qu'élogieux, pour vanter les qualités civiles si ce n'est physiques ou littéraires - du défunt Qiao Xuan (110-184), qui possédait « la force majestueuse du tigre rugissant, la culture florissante du dragon ciselé (wen fan diaolong) » ${ }^{64}$. Cai Yong appariait ainsi les deux vertus complémentaires du wen (culture civile, politique) et du $w u$ (militaire). Au début des Wei septentrionaux, Gao Yun (390-487) ${ }^{65}$ jouait à son tour du dragon ciselé, mais en associant cette fois le wen à son autre complément naturel - l'essence ou la simplicité (zhi). Il s'agissait encore d'un éloge, composé par Gao Yun sur ses vieux jours (après 466) en l'honneur des éminents lettrés sans fonction qui, autrefois, avaient été comme lui appelés au service de l'empire ${ }^{66}$. Il réservait à son oncle les plus beaux clichés :

Ses connaissances vastes et exhaustives, son entendement des [choses] inédites et singulières proviennent du fond de son cœur. [Chez lui,] l'essence

64 "Taiwei Qiao Xuan beiyin » (Inscription pour l'envers de la stèle du ministre des armées Qiao Xuan), Quan Hou Han wen (Prose complète des Han postérieurs, in Yan Kejun [1762-1843], Quan Shanggu Sandai Qin Han Sanguo Liuchao wen, Pékin, Zhonghua shuju, rééd. 1991, vol. 1), juan 77, p. 3a.

65 Poète, littérateur, historien et commentateur des classiques originaire de Bohai, Gao Yun fut appelé au service de l'empire en 431, et devint un homme d'État particulièrement influent à la fin de sa vie, après qu'il eut fait assassiner un ministre en 466 (cf. Wei shu [Livre des Wei septentrionaux], juan 48, p. 10671090).

$66 \mathrm{Cf}$. «Zhengshi song » (Éloge des lettrés appelés [au service de l'empire]), cité par la biographie de Gao Yun dans le Wei shu, juan 48, p. 1078-1085. En 431, l'empereur Taiwu (r. 423-452) des Wei septentrionaux sacrifiait à cette coutume éminemment politique en menant une vaste campagne de recrutement parmi plusieurs centaines de lettrés retirés, appelés à servir à diverses fonctions (cf. ibid., juan 4, p. 79). Gao Yun devint alors docteur auprès du secrétariat impérial. Il explique dans l'introduction de son éloge — limité aux trente-quatre personnes qui répondirent avec lui à l'appel de l'empereur - que la Cour était à cette époque " pleine de jeunes gloires montantes (ou arrivistes, houjin zhi shi), tandis que résidaient dans les campagnes des gens qui n'etaient pas des hommes du passé ", et qui pourtant « n'avaient nul endroit (objet) où confier leur esprit » (wu jixin zhi suo ; ibid., juan 48, p. 1081). 


\section{Valérie Lavoix}

est l'égale du jade de He ; l'ornement (la culture) a la splendeur du dragon ciselé (wen bing diaolong) ${ }^{67}$.

Mais on ne peut être certain que l'auteur vantait les qualités de la substance et de l'ornement dans les œuvres de son oncle.

À la même époque que Gao Yun, deux littérateurs célèbres employaient - en se référant au Shiji - la métaphore du dragon ciselé dans un sens plus précisément littéraire. Dans l'autobiographie déguisée que constitue son "Miaode xiansheng zhuan " (Biographie de monsieur Vertu Merveilleuse), Yuan Can (420-477) se décrivait ainsi :

Enfant, il fut souvent malade, d'un tempérament paresseux et négligent ; il était sans désir ni dessein. Pourtant, il connaissait les idées essentielles de tous les discours des cent écoles et des neuf traditions, ainsi que les arts du graveur de dragons et du spécialiste du ciel - mais il n'en devint pas célèbre pour autant. ${ }^{68}$

En invoquant les surnoms de Zou Shi et Zou Yan, Yuan Can ne prétendait pas posséder les techniques de la tradition yinyang, car il avait auparavant nommé les neuf traditions; il donnait de fait à la gravure de dragons et à la dissertation sur le ciel les sens élargis de littérature et de discours : monsieur Vertu Merveilleuse possédait l'art littéraire comme celui de l'éloquence.

Vers 475, le poète Jiang Yan (444-505) sollicitait encore davantage les surnoms des maîtres des Royaumes combattants : il semblait attribuer à Zou Yan la réputation de Zou Shi, en qualifiant de gravure de dragons le talent de disserter (bian; éloquence, ou forme littéraire de l'argument) on se souvient que Zou Yan « dissertait avec ampleur [des sujets] les plus vastes et lointains " ${ }^{69}$. Le « Bie fu » (Fu sur la séparation) se conclut en tout cas ainsi :

67 Wei shu, juan 48, p. 1084. Le Beishi (Histoire [des dynasties] du Nord ; juan 31 , p. 1127) cite le même texte avec la variante wen zhao diaolong.

68 Song shu (Livre des [Liu] Song), juan 89, p. 2230.

69 Dans la préface aux biographies de gens de lettres du Wei shu («Wenyuan liezhuan », juan 85, p. 1869), on trouve une confusion plus flagrante encore : c'est au beau parleur Chunyu Kun, la « corne d'abondance » du Shiji (cf. supra, 
[Même s'il possédait] par ses $f u$ la réputation de chevaucher les nuages ${ }^{70}$, ou par ses arguments la renommée du graveur de dragons, qui pourrait décrire la physionomie d'un départ soudain, dépeindre le sentiment d'un adieu éternel ? ${ }^{71}$

Appariée à la métaphore de la « chevauchée des nuages », celle du dragon ciselé était définitivement consacrée comme image de l'art de composer une cuvre littéraire particulièrement accomplie.

C'est à ce titre que l'expression se spécialisa pour désigner le talent dont héritent les meilleurs littérateurs. Dans la clausule, éloge final de la biographie de Cui Yin (?-92 ap. J.-C.), savant et poète dont la renommée fut un temps associée à celle de Ban Gu (32-92), et qui composa notamment un «Éloge des quatre orients » («Sixun song ») dont « l'expression était extrêmement belle et raffinée ${ }^{72}$, Fan Ye (398-445) écrivait :

Les Cui étaient des maîtres des belles-lettres, qui de génération en génération se transmirent [l'art] de graver les dragons (shi shan diaolong). ${ }^{73}$

De fait, le fils de Cui Yin, Cui Yuan (78-143), était éminemment doué pour la composition littéraire, et excellait dans des genres très variés ${ }^{74}$. Son

n. 55), qu'est attribué le talent de Zou Shi : «Quand Chunyu Kun quitta le pays de $\mathrm{Qi}$, il portait le titre de graveur de dragons. » Cette référence est appariée à une évocation du génie de Qu Yuan, et, dans le contexte du «jardin des lettres », la gravure de dragons désigne manifestement l'art littéraire le plus accompli.

70 Lorsque Sima Xiangru présenta son « Daren fu » (Fu sur le grand homme, ou « Daren zhi song », Éloge du grand homme) à l'empereur Wu des Han, « le fils du ciel en fut enchanté ; tourbillonnant dans les airs il avait l'impression de chevaucher les nuages » (Shiji, juan 117, p. 3063 ; cf. Y. Hervouet, Le chapitre 117 du Che-ki (Biographie de Sseu-ma Siang-jou), Paris, p. 204 ( « Bibliothèque de l'IHEC », XXIII). « Chevaucher les nuages » est devenu une métaphore stéréotypée du sublime talent de Sima Xiangru, et plus généralement de la réussite des plus belles expositions $(f u)$.

71 Wenxuan, juan 16, p. 31b.

72 Hou Han shu, juan 52, p. 1718.

73 Ibid., p. 1733.

74 Cf. $i b i d$, p. 1724. 
petit-fils, Cui Shi (?-ca 168), lui aussi fort talentueux, laissa de nombreuses œuvres poétiques et prosaïques ${ }^{75}$; le cousin de Cui Shi, Cui Lie (d.i.), était encore doté d'un beau talent de littérateur ${ }^{76}$.

Sous les Liang, le présomptueux Wang Jun (481-549), piqué de jalousie, revendiqua haut et fort pour sa propre famille le privilège de s'être « de génération en génération transmis [l'art] de graver les dragons »- estimant que les mérites du clan des Cui en cette matière n'étaient rien encore comparés à ceux des Wang ${ }^{77}$. Le biographe du clan des Liu de Pengcheng, qui compta tant de poètes fameux, écrirait à son tour que « [Liu] Hui (458502 ) transmit à tous ses fils [1'art] de graver les dragons ${ }^{78}$. En 501, dans l'ordonnance qu'il composa à la demande de l'impératrice Xuande (455$512)^{79}$ afin d'exhorter le futur empereur Wu (Xiao Yan ; 464-549) à accepter le titre de duc de Liang, Ren Fang (460-508) avait utilisé une tournure comparable pour vanter le talent littéraire du candidat au trône :

75 Cf. ibid, p. 1725.

76 Cf. ibid, p. 1732.

77 Cf. la lettre de Wang Jun à ses fils, citée par le Liang shu (juan 33, p. 486) : "L'histoire nous dit que le clan des Cui de Anping comme celui des Ying de Runan virent se succéder les talents littéraires sur plusieurs générations, et c'est pourquoi Fan Weizong (Fan Ye) écrivait que la famille Cui "de génération en génération se transmit [1'art de] graver les dragons". Mais il n'y eut chez ceux-là que deux à trois générations de père en fils ; ils n'ont point comme en notre maison partagé gloire, titres et statut durant sept générations, chacun ayant d'ailleurs [mérité] le recueil individuel [de ses œuvres]. [Shen Yue luimême disait] "Jamais il n'y eut si belle continuité dans la réussite mandarinale, si longue succession de talents littéraires que chez le prospère clan des Wang." »

78 Nanshi, juan 39, p. 1015. Parmi les littérateurs reconnus du clan des Liu, on doit naturellement citer Liu Xiaochuo (481-539), mais aussi Liu Xiaoyi, autrement appelé Liu Qian (484-550), ou Liu Xiaowei (490?-549), de même que Liu Bao (482-511), Liu Ru (485-543) ou Liu Zun (488-535).

79 Après avoir été concubine du prince héritier Wenhui (458-493) des Qi, la future impératrice Xuande devint l'année suivante concubine principale du fils de Wenhui, le prince de Yuling (473-494). Celui-ci ne régna que durant douze mois (en 493-494), mais elle conquit grâce à lui le titre d'impératrice. Elle quitta le palais sous le règne de l'empereur Mingdi (r. 494-498), pour y revenir en 501, sur l'invitation du futur empereur Wu des Liang (cf. Nan Qi shu [Livre des Qi du Sud], juan 20, p. 392). 
Quant aux belles-lettres, [vous] avez reçu [le talent] de graver les dragons (wen shan diaolong); de chaque texte achevé le brouillon est détruit aussitôt. ${ }^{80}$

Un siècle plus tard, le dragon ciselé était une expression consacrée de plus en plus souvent employée dans des discours généraux ou historiques sur la littérature, pour désigner le talent littéraire, la beauté d'une œuvre ou du style ${ }^{81}$. L'homme de lettres Wang Zhen (?-605) en usa lorsqu'en 604 il voulut gagner l'estime d'un prince en lui présentant son recueil d'œuvres personnel (Wenji). Dans une sorte de condensé d'histoire de la littérature antique, il rattachait - tout comme Liu Xie — la gravure des dragons à l'auguste enseignement de Confucius :

Le bel accomplissement (wenzhang) de Confucius s'accordait par sa nature même à la voie du ciel. Il transmit son idéal exemplaire à [Zi]you et [Zi]xia ; les dernières ondes en résonnent encore chez Qu [Yuan] et Song [Yu] : des dragons ciselés notre héritage tout entier se trouve dans les « Airs» [du Shijing $]$ et le « [Li] sao ». ${ }^{82}$

La gravure de dragons avait bel et bien perdu tout caractère de futilité, et la métaphore n'avait plus depuis longtemps la nuance péjorative qu'on pouvait

80 «Xuande huanghou ling» (Ordonnance de l'impératrice Xuande), Wenxuan, juan 36, p. $2 \mathrm{~b}$.

81 Les historiens du début des Tang aimaient particulièrement employer cette métaphore. Vers 630, les auteurs du Zhou shu (Livre des Zhou, juan 38, p. 690) disaient de Su Liang (?-551) et d'autres savants et littérateurs de sa génération qu'ils « avaient pour les belles-lettres reçu [le talent] de graver les dragons ». La préface des biographies de gens de lettres du Bei Qi shu (Livre des Qi septentrionaux, «Wenyuan liezhuan », juan 45, p. 601), achevé en 636, dit des poètes et hommes de talent qui succédèrent à ceux des Han qu'ils « laissaient libre cours aux précieuses beautés des dragons ciselés. » Selon la préface des "Wenyuan liezhuan » du Jin shu (Livre des Jin ; juan 92, p. 2369), achevé en 648 , ce sont Ban Gu et Zhang Heng (78-139) qui « sur la soie et les tablettes [d'écriture] firent naître les dragons ciselés. "

82 Sui shu, juan 76, p. 1737. Le Recueil des ceuvres de Wang Zhen fut fort apprécié, et son auteur reçut en récompense quatre bons chevaux. 


\section{Valérie Lavoix}

(ou non) soupçonner dans le surnom de Zou Shi. Sans doute allait-elle déplaire bientôt : elle disparaît en tout cas de la littérature des annales littérature qui n'est pas seulement historique, grâce aux cuvres, lettres, mémoires ou préfaces, dont elle livre souvent l'intégralité - après le milieu du viI ${ }^{\mathbf{e}}$ siècle ${ }^{83}$. Le Wenxin diaolong avait jusque-là connu sa plus grande faveur (en Chine tout au moins, avant le milieu du XVI ${ }^{e}$ siècle) : il a évidemment grandement contribué à la vogue de la métaphore des dragons ciselés entre 500 et 650 .

Pour achever de démontrer la valeur de l'emblème figurant dans le titre du Wenxin diaolong, il faut encore rappeler que, depuis les Han, tandis que les dragons ciselés s'installaient dans le vocabulaire de l'éloge littéraire en conquérant leurs titres de noblesse, leur pendant ou antonyme naturel on sait que les dragons appartiennent à cette famille - les vermisseaux ciselés (diaochong), connaissait une fortune presque aussi grande : on ne peut imaginer que ces deux expressions n'aient pas résonné l'une par rapport à l'autre.

Cette autre métaphore fut inventée par Yang Xiong, et s'appliquait tout d'abord exclusivement au fait de composer des $f u$ :

Quelqu'un m'a demandé : «Avez-vous aimé composer des $f u$ dans votre jeunesse ?» J'ai répondu : « En effet, comme les enfants s'exercent à graver sceaux et vermisseaux », pour ajouter l'instant d'après : « Un homme mûr ne s'y adonne point. ${ }^{84}$

Graver des vermisseaux et sculpter des sceaux (diaochong zhuanke) signifiait au sens propre s'exercer à tracer des caractères selon les deux styles vermiforme ( «têtard », chongshu) et sigillaire (des sceaux et tablettes, $k e f u)$ - parmi les six formes d'usage dites de Qin. Yang Xiong comparait

83 Grâce à l'outil étonnant que constitue la concordance électronique des vingtcinq histoires dynastiques, on s'aperçoit que les dernières occurrences de la métaphore diaolong sont celles que j'ai citées ci-dessus, dans la note 81 . L'expression ne désigne plus ensuite au sens premier que des insignes ou emblèmes gravés de dragons.

84 Fayan, «Wuzi », p. 4. 
le $f u$ à un exercice ou à un amusement qui aurait pu être considéré comme formateur (puisque les enfants s'y adonnent), mais qui lui parut, arrivé à l'âge mûr, inconséquent ou même dangereux :

On me dit : « Le fu ne peut-il servir à insinuer une critique (feng) ? » « S'il y a critique, il faut qu'elle fasse cesser [la faute]. Si elle n'y parvient pas, je crains qu'elle ne l'encourage inévitablement. " ${ }^{85}$

Ainsi, Sima Xiangru présenta le «Fu sur le grand homme » à l'empereur Wu des Han pour critiquer la passion invétérée que vouait son souverain aux immortels. Pourtant, il manqua lamentablement son but, car l'empereur pensa plus que jamais à «s'élever dans les airs pour chevaucher les nuages. Où l'on voit clairement que le fu encourage plutôt qu'il n'empêche [la faute]. " 86

Des exercices de calligraphie, on oublia bientôt la nature technique, pour ne garder que l'image de la gravure de vermisseaux : parfois, celle-ci continua de désigner spécialement l'art du $f u$, ou plus largement celui de la composition poétique, en portant une valeur neutre ou laudative ${ }^{87}$. Mais le

$85 \mathrm{Ibid}$. Yang Xiong condamnait ensuite le fu par le biais d'une seconde analogie : « On me dit : "La gaze de brume n'est-elle pas la plus belle des étoffes?" "C'est la plus grande nuisance de l'ouvrage féminin" [...] Le fu est beau mais nuit à la rectitude, car il est beau par la licence. »

86 Cf. supra note 70 et la biographie de Yang Xiong dans le Han shu, juan 87 « xia », p. 3575.

87 Il y eut sous les Wei et les Qi septentrionaux un excellent poète dont le talent fut comparé à celui de Wang Can (177-217) : «À partir du règne de l'empereur Xiaoming (r. 515-528) des Wei septentrionaux, les belles-lettres connurent un grand essor, et la beauté des vermisseaux ciselés de [Xing] Shao (496-?) fit de lui la célébrité de son époque. Aussitôt que chacune de ses œuvres était publiée, le prix du papier augmentait sur les marchés de la capitale ; on la récitait partout aux alentours et au lointain » (Bei Qi shu, juan 36, p. 476). Lorsque le célèbre littérateur des Qi septentrionaux Xue Daoheng (540-609), devenu diplomate sous les Sui, fut envoyé en ambassade chez les Chen, il est dit : «Au Sud, on aimait beaucoup la poésie (pianshi), et le souverain des Chen était particulièrement féru de vermisseaux ciselés. Chaque fois que [Xue] Daoheng composait quelque chose, les gens du Sud le déclamaient tous en chœur » (Sui shu, juan 57, p. 1406). Cf. également le Jiu Tang shu (Ancien livre des Tang ; 
plus souvent, elle servit à condamner les œuvres littéraires considérées comme trop ornées, artificieuses ou futiles, ou le fait de composer de la poésie comme une occupation vaine, frivole, voire indigne : on fustigea l'« art mineur de la gravure de vermisseaux" (diaochong xiaoyi ou diaochong xiaoji). On vit même certains officiers en être accusés officiellement comme d'un crime, comme en 499, dans le décret impérial ordonnant l'emprisonnement du poète Xie Tiao (464-499). Parce qu'il avait refusé de soutenir le complot de Xiao Yaoguang, prince de Shi'an (468499), qui rêvait de renverser le marquis Donghun (r. 498-501) des Qi ${ }^{88}$,

juan 190 « shang », p. 4982), au sujet des æuvres poétiques de Wang Wei (699761) et $\mathrm{Du} \mathrm{Fu}(712-770)$. On trouve par ailleurs recensé dans le traité bibliographique du Xin Tang shu (Nouveau livre des Tang, juan 60, p. 1603), parmi les recueils personnels, un Diaochong $j i$ (Recueil de vermisseaux ciselés) en un juan, composé par Wang Zhu (vII siècle). Ce titre succède immédiatement au Recueil des æuvres de Wang Wei en dix juan, et pourrait avoir désigné des pièces poétiques. Quoi qu'il en soit, les occurrences neutres ou laudatives du composé diaochong dans les histoires dynastiques sont toutes assez tardives, comme s'il avait fini par remplacer la gravure de dragons.

88 Ces deux personnages sont un parfait prétexte pour me permettre de rappeler quelle était l'atmosphère politique à la Cour des Qi du Sud, alors que les gens de lettres et la bonne société s'occupaient si bien de ciseler dragons et vermisseaux et que Liu Xie composait le Wenxin diaolong. Neveu de l'empereur Ming des Qi, Xiao Yaoguang avait été son plus fidèle conseiller et son grand complice en matière d'assassinats politiques, notamment à l'époque des complots et destitutions successives au prix desquels Mingdi monta sur le trône en 494. Ils organisèrent ensemble la tuerie des princes des Qi au cours de la même année, puis, au premier mois de 498 , celle des descendants des empereurs Gao (r. 479-482) et Wu (r. 482-493) qui avaient survécu à la première hécatombe. Lorsque son cousin, le fils de l'empereur Ming et non moins funeste marquis Donghun (du « crépuscule à l'Orient »), fut monté sur le trône, Xiao Yaoguang complota pour le renverser, prit les armes, et finit décapité (cf. Nan Qi shu, juan 45, pp. 788-91). Le règne de Donghun fut ponctué par la répression sanglante de nombreux autres complots : devenu empereur à l'âge de quinze ans, d'une méfiance décrite comme paranoïaque et justifiée à la fois, le jeune et cruel souverain s'entourait d'une garde prétorienne, et faisait évacuer, à plusieurs dizaines de $l i$ à la ronde, toutes les habitations qui se trouvaient sur son chemin lorsqu'il se déplaçait. Les riches demeures ainsi désertées étaient 
Xie Tiao fut gravement calomnié auprès du souverain, à qui l'on demanda sa tête. Convaincu par les calomniateurs, Donghun argumenta sa condamnation en ces termes : «Il ne cesse de tourner en dérision l'habit d'officier en s'adonnant à l'art négligeable de la gravure de vermisseaux (diaochong boji). " ${ }^{89}$ Et Xie Tiao mourut en prison.

$\mathrm{Ce}$ cas est sans doute l'un des plus dramatiques qu'on puisse rencontrer ${ }^{90}$. Car, à l'instar des dragons ciselés, la métaphore des vermisseaux fut employée assez souvent dans des textes polémiques, historiques ou politiques - apparemment - moins sulfureux. Et tandis que les dragons paraissaient de plus en plus souvent à partir des années 470 , c'est entre les années 495 et 515 que les vermisseaux se seraient multipliés.

Lorsque Shen Yue (441-513) répondit à la fameuse lettre de Lu Jue (472-499) qui attaquait sa théorie des «quatre tons ${ }^{91}$, il semble avoir

pillées, et parmi maints détails anecdotiques rapportés par les historiens, un malade abandonné au bord d'une rivière y fut noyé la tête inondée de boue afin que sa mort fût plus rapide ; une femme sur le point d'accoucher qui n'avait pu quitter sa maison fut éventrée afin que Donghun pût savoir si l'enfant était une fille ou un garçon ; un vieux moine trop malade pour quitter son monastère fut découvert dans sa cachette et transpercé de cent flèches comme un vulgaire daim (cf. Nan Qi shu, juan 7, p. 97-108, et Nanshi, juan 5, p. 152-153).

89 Nan Qi shu, juan 47, p. 827.

90 Lorsque Yan Yanzhi (384-456) fut démis de ses fonctions pour malhonnêteté dans les années 440 , le mémoire qui fut présenté contre lui à la chancellerie l'accusait entre autres excès et inconduites d' $*$ aimer les vermisseaux ciselés sans jamais vouloir y renoncer » (Song shu, juan 73, p. 1902) : la punition fut moins sévère, mais il n'en s'agissait pas moins d'une dénonciation officielle.

91 Entre 494 et 499 ; cf. sur ce sujet F. Tökei, " Textes prosodiques chinois au début $\mathrm{du} \mathrm{vl}^{\mathrm{e}}$ siècle », in Mélanges de sinologie offerts à Monsieur Paul Demiéville, Paris, Institut des hautes Études Chinoises, vol. 2, 1974 («Bibliothèque de l'Institut des Hautes Études Chinoises », XX), p. 297-312 ; l'ensemble du cinquième chapitre ( "The Flowering of the Yung-ming Style ») de l'ouvrage de R. Mather, The Poet Shen Yüeh (441-513), The Reticent marquis, Princeton, Princeton University Press, 1988, p. 37-84 ; F. Martin, « Note sur la série des quatre tons », Extrême-Orient Extrême-Occident, 12, p. 67-78; V.H. Mair et Tsu-lin Mei, « The Sanskrit Origins of Recent Style Prosody », HJAS, 51 (2), p. 396-401. 


\section{Valérie Lavoix}

voulu récupérer l'ancienne condamnation du fu par Yang Xiong en la détournant de son but premier. Afin de justifier le fait que les sages du passé n'avaient pas ressenti le besoin d'utiliser l'art des tons et rimes pour transmettre leur enseignement, il écrivait :

Ainsi, par exemple, Ziyun (Yang Xiong) comparait cela à « la gravure de sceaux et vermisseaux » en ajoutant que « les hommes mûrs ne s'y adonnent point $»{ }^{92}$

Sans doute voulait-il ainsi couper l'herbe sous le pied des critiques du contrepoint tonal, en lui appliquant - avant eux - la moquerie des vermisseaux gravés; il ne s'en laissait pas moins toute latitude pour légitimer l'art des tons et rimes dans le domaine poétique, et non pas moral ou didactique.

Sous les Liang, Shen Yue usait encore de la métaphore, mais dans un sens plus général et, selon l'occasion, en sollicitant ou réfutant sa valeur dépréciative. Révolté par le nombre pléthorique des officiers que comptait la capitale (plusieurs dizaines de milliers selon lui), il expliqua un jour que les moyens de sélection étaient mauvais et la fonction publique d'un accès beaucoup trop facile :

S'il suffit de répondre à cinq questions pour être un talent remarquable (xiucai; gradué du premier rang) et de composer une seule dissertation d'examen pour devenir pieux et intègre (xiaolian ; gradué du second rang), c'est vraiment [se fier au] aux méthodes mineures des vermisseaux ciselés

92 Nan Qi shu, juan 52, p. 900 . Shen Yue aurait dès 487-488 employé pour son compte la métaphore des vermisseaux dans un sens péjoratif. Dans une biographie du Song shu (juan 55, p. 1552), le jugement de l'annaliste dit: «Depuis que les Wei (des Trois Royaumes) ont reçu le mandat, les souverains sont férus de la gravure de vermisseaux, et les écoles dédaignent [les commentaires] par phrases et paragraphes. " Cette phrase étonne quelque peu sous la plume de Shen Yue : je ne sais si elle peut être attribuée à l'un de ses prédécesseurs dans la rédaction des soixante-dix fascicules d'annales et biographies du Song shu auxquels Shen Yue mit la dernière main en 487-488, dans l'espace d'une année à peine. 
(diaochong xiaodao), au lieu de rechercher la réussite de l'administration. Sélectionner les talents de cette manière n'est que pure mascarade ${ }^{93}$

Mais quand il rédigea une préface pour le recueil personnel des œuvres de l'empereur Wu des Liang (r. 502-549), il se fit l'avocat de l'ornement en littérature : « L'art mineur des vermisseaux ciselés ne nuit en rien à la grande méthode. " 94

Quelques années plus tard, Zhong Rong (469?-518) employa également l'image de la gravure de vermisseaux : on la trouve deux fois dans le Shipin (Classification des poètes), et significativement dans deux contextes distincts - général et particulier, comme on vient de le voir chez Shen Yue. Dans la notice consacrée à Wang Jian (452-489), Zhong Rong suppose que cet auteur considérait la poésie comme une occupation futile : « Il avait d'ambitieux desseins quant au gouvernement de l'empire ; sans doute méprisait-il [la poésie] comme gravure de vermisseaux. ${ }^{95}$ Et ce jugement laconique suffit à expliquer que Wang Jian soit classé dans le rang inférieur du Shipin. Il n'en va pas de même avec Ruan Ji (210-263), qui ne méprisait assurément pas l'art poétique, mais la poésie trop ornée ou artificieuse : « Il ne s'efforçait point aux vermisseaux ciselés. » 96

Dans son sens général - la gravure de vermisseaux comme art, procédé ou occupation futiles -, on verra l'expression diaochong servir souvent dans des exhortations au sérieux adressées à l'empereur ou au prince héritier ${ }^{97}$. Dans son sens propre - un art littéraire ou poétique dénoncé

93 Tongdian (Histoire générale des institutions, Pékin, Zhonghua shuju, 1988); "Xuanju » 4, juan 16, p. 388.

94 Autrement dit au bon gouvernement de l'empire ; «Liang Wudi ji xu »; Yiwen leiju (Somme analytique des arts et des lettres, Shanghai, Shanghai guji chubanshe, 1999), juan 14, p. 269.

95 Shipin, « xia », Chan Hing-ho, Zhong Rong Shipin jijiao, Paris/Hong Kong, Centre de publication Asie orientale, 978, p. 81, 11. 22-3.

96 Shipin, «shang », p. 69, 1. 12.

$97 \mathrm{Li} \mathrm{E} \mathrm{(d.i.)} \mathrm{adressa} \mathrm{un} \mathrm{mémoire} \mathrm{resté} \mathrm{célèbre} \mathrm{à} \mathrm{l'empereur} \mathrm{Wen} \mathrm{(r.} \mathrm{581-604),}$ fondateur des Sui, pour lui déconseiller de suivre l'exemple funeste des souverains de Wei, à l'époque des Trois Royaumes, qui, « férus de poésie, négligèrent la grande doctrine du gouvernement et prisèrent l'art mineur de la 


\section{Valérie Lavoix}

comme artificieux et vain -, elle se spécialisera dans un autre emploi : les mémoires adressés au trône l'utiliseront pour critiquer le mode de recrutement des officiers, et se lamenter que l'on accorde en cette matière trop d'importance au talent littéraire, et plus particulièrement poétique ${ }^{98}$. Le plus célèbre auteur à avoir illustré - et peut-être inauguré - cette seconde tradition est Pei Ziye (469-530), dans ce qu'on a appelé l'essai sur la gravure de vermisseaux, le « Diaochong lun».

La date et la nature de ce texte sont un double sujet de controverse, dont la solution est sans doute en réalité unique. Les savants les plus éminents considèrent qu'il fut composé entre 527 et $530^{99}$, dans le contexte d'une polémique entre les tenants d'une conception didactique de la littérature - prônant le retour à la simplicité antique et dont Pei Ziye fut le plus célèbre représentant - et les défenseurs de la littérature nouvelle, autrement dit de la poésie ornée, galante, qui connut son apogée dans le style du

gravure de vermisseaux » (Sui shu, juan 66, p. 1544). Dans un mémoire au trône pour exhorter le prince héritier à l'étude et au sérieux, Liu Ji (?-645) lui reprochait de «soudain négliger les affaires importantes pour aussitôt porter [son attention] aux vermisseaux ciselés » (Jiu Tang shu, juan 74, p. 2610). En 639, Zhang Xuansu (?-644) faisait de même : « Et quant aux arts mineurs de la gravure de vermisseaux, il ne faut s'y adonner que de temps à autre, en remplacement des jeux de tablettes et d'échecs » (ibid., juan 75, p. 2642).

98 Sous les Tang, après le milieu du vir ${ }^{\mathrm{e}}$ siècle, on cessa de faire l'éloge des dragons ciselés ; simultanément, on se plaignit souvent de l'importance accordée à la gravure de vermisseaux - plutôt qu'aux vertus d'intégrité et de sobriété pour sélectionner les talents depuis les Dynasties du Sud (cf. par exemple Jiu Tang shu, juan 101, p. 3137, et juan 166, p. 4359).

99 Cf. notamment Luo Genze, Zhongguo wenxue piping shi, Shanghai, Shanghai gudian wenxue chubanshe, 1957, vol. 1, p. 135. La plus ancienne édition du «Diaochong lun » est celle du Tongdian (achevé en 801 par Du You [735812], « Xuanju » 4, juan 16, p. 389-390), où le texte est précédé par un autre développement de Pei Ziye qui se trouve lui-même introduit par les mots : «L'annonceur aux audiences Pei Ziye fit également le jugement suivant [...] » (ibid., p. 388). Pei Ziye fut nommé annonceur aux audiences en 527 et mourut en 530, mais il est probable qu'on lui a ici donné ce titre - selon l'usage traditionnel - parce que c'était le dernier ou le plus important qu'il ait jamais occupé. 
palais ${ }^{100}$ et le Yutai xinyong (Nouveaux chants de la terrasse de jade). Le «Diaochong lun» serait un pamphlet visant directement les poètes «modernes », cette hypothèse étant confortée par le fait que Xiao Gang (503-551), futur empereur Jianwen des Liang, et sous le patronage duquel le style du palais fut mis à l'honneur, se moqua vertement de Pei Ziye dans sa «Lettre au prince de Xiangdong » ${ }^{101}$. Néanmoins, selon les conclusions d'une étude de Uchida Shinnosuke ${ }^{102}$, et en s'appuyant tout particulièrement sur la comparaison du « Diaochong lun » avec d'autres textes de Pei Ziye conservés dans les mêmes conditions ${ }^{103}$, on doit il me semble considérer que ce texte est issu du Song lüe (Abrégé [d'histoire] des Song) composé par Pei Ziye à la fin des Qi ${ }^{104}$ — autrement dit en même temps que le Wenxin diaolong - qu'il s'insérait sans doute dans un développement

100 Gongti, style en faveur au palais du prince héritier de 531 à 549 , lorsqu'il fut occupé par Xiao Gang, sous l'égide duquel fut compilé le Yutai xinyong.

101 Cf. supra, note 10.

102 Article traduit en chinois par Chen Xizhong et révisé par Zhou Yilang, sous le titre «Pei Ziye "Diaochong lun" kaozheng" (Critique des sources du «Diaochong lun» de Pei Ziye), Gudai wenxue lilun yanjiu, 6, Shanghai, Shanghai guji chubanshe, 1981, p. 231-250.

103 Le Tongdian contient - hormis le « Diaochong lun » - deux textes de Pei Ziye introduits par le même mot lun (jugement, au sens des commentaires ajoutés par les historiens à la fin des annales, biographies ou monographies), et qui traitent tous deux du problème de la sélection des officiers sous les Liu Song (cf. Tongdian, juan 14, p. 334, et juan 16, p. 388-389). Ils sont également cités par le Zizhi tongjian (Miroir universel à l'usage du bon gouvernement), qui n'aurait utilisé de Pei Ziye que le seul Song lüe (cf. l'article de Uchida Shinnosuke, cité dans la note précédente, p. 238-239). Rien ne distingue formellement le «Diaochong lun » de ces deux textes, et l'on y trouve énumérées successivement deux ères des Liu Song (voir ci-dessous).

104 Si l'on se fie notamment à une phrase de Pei Ziye citée par le Jiankang shilu (Documents authentiques sur Jiankang, compilés en 756 par Xu Song [d.i.], Pékin, Zhonghua shuju, 1986, juan 14, p. 558), l'historien aurait composé cet ouvrage parce qu'il était désœuvré à cette époque. Il le fit en tout cas après la publication du Song shu (en 488), qu'il voulait compléter et corriger sur certains points. L'ouvrage comptait vingt juan et fut perdu. 


\section{Valérie Lavoix}

sur les problèmes du recrutement des officiers, et ne portait peut-être pas à l'origine le titre qu'on lui connaît depuis la fin du $x^{\mathrm{e}}$ siècle ${ }^{105}$.

Le « Diaochong lun » ressemble en effet beaucoup à ces développements sur l'histoire de la littérature que l'on trouve abondamment dans les histoires dynastiques en introduction ou conclusion des biographies collectives de gens de lettres ou de lettrés, en commentaire ou postface à la biographie de tel ou tel littérateur, aux annales d'un empereur, ou encore au sein des traités bibliographiques, sur les rites, les officiers ou la musique. À partir des Six Dynasties, ce genre de développement se fit de plus en plus fréquent pour décrire l'essor sans précédent des activités littéraires durant cette période et pour expliquer la décadence politique, économique, sociale, institutionnelle que les historiens postérieurs ( $\mathrm{au} \mathrm{vI} \mathrm{I}^{\mathrm{e}}$ et surtout au viI ${ }^{\mathrm{e}}$ siècle) affectèrent de dénoncer.

L'essai - si ce n'est un jugement de l'historien — de Pei Ziye commence ainsi par un survol de l'histoire de la poésie depuis le Shijing jusqu'aux Jin orientaux, se concentre sur l'époque des Liu Song jusqu'à l'ère Daming (457-465), avant de poursuivre avec un développement sur la période postérieure : celle-ci peut très naturellement être la dynastie des Qi du Sud, sans forcément s'étendre jusqu'aux années 527-530 des Liang ${ }^{106}$. Selon Pei Ziye, le modèle parfait de la poésie est le Shijing, que la propension des poètes à embellir et orner profusément leurs vers ne cessa de faire déchoir toujours plus - à partir des Chuci. Après Sima Xiangru, les « expositions $(f u)$, poèmes, chants et éloges » s'amassèrent en nombre considérable :

105 Le « Diaochong lun » figure pour la première fois avec ce titre dans la section «Lunwen » (De la littérature) du Wenyuan yinghua (Florilège du jardin des lettres), achevé en 986 sous la direction de Li Fang (925-996). Cette édition comporte quelques variantes par rapport au texte du Tongdian, qui le précède de près de deux siècles et paraît plus fiable en certains points.

$106 \mathrm{~J}$ 'interprète ce développement comme une digression au présent visant à souligner a fortiori la gravité du mauvais tournant pris par les hommes des Liu Song vers $460:$ la fin du texte clôt cette digression en nommant à nouveau les Liu Song, dont la déchéance a été assez démontrée pour pouvoir conclure sur le thème rebattu des États en perdition (cf. Tongdian, juan 16, p. 390). 
Cai Yong compara cela à des bouffonneries ${ }^{107}$, et Yang Xiong regretta [de s'y être adonné] comme à un enfantillage. ${ }^{108}$

Les choses ne s'arrangèrent pas vraiment par la suite. Pourtant :

Du début des [Liu] Song jusqu'à l'ère Yuanjia (424-454), on s'occupa beaucoup des Classiques et de l'histoire. [Mais] la génération de l'ère Daming se prit de passion pour ce genre de littérature. Les talents éminents composaient des rimes rares et ne faisaient plus cas des génies du passé : cette vogue gagna toute l'époque, et il en alla de mal en pis. Depuis lors, les jeunes gens des villages comme les cadets de la noblesse rejettent tous unanimement les Six Classiques pour « déclamer leur sentiment personnel " (yinyong qingxing) ${ }^{109}$. Les savants ne s'occupent plus que de multiplier les métaphores (boyi) ${ }^{110}$ et déclarent que [les commentaires] par phrases et paragraphes sont laborieux et obtus. Par un ornement outrancier [les poètes] ruinent le modèle canonique, et l'élégance formelle leur tient lieu de profession ; ils dédaignent les vents et les cordes tout comme ils font fi des rites et de la rectitude (feizhi hu liyi) ${ }^{111}$. Leur motivation [la plus] profonde est de vénérer herbes et arbres, leur aspiration [la plus] élevée s'arrête au vent et aux nuages ${ }^{112}$; vaine est leur ardeur et faible leur intention. Ils sont habiles sans être pertinents, obscurs mais dénués de profondeur : c'est assurément de l'influence des [Liu] Song que [ces poètes] sont les héritiers. ${ }^{113}$

$107 \mathrm{Cf}$. la cinquième des sept propositions de politique soumises par Cai Yong en 177 à l'empereur Ling (r. 168-189) des Han postérieurs; Hou Han shu, juan 60 « xia », p. 1996 (d'après Uchida Shinnosuke, article cité, p. 241).

108 Tongdian, juan 16, p. 389. Il est ensuite question du poème pentasyllabique sous les Han, Wei et Jin.

109 Cf. la « Grande préface» du Shijing, « Daxu », Shisanjing zhushu, p. 271c.

110 Cf. $L i j i$, «Xueji », p. 1522b.

111 Cf. «Daxu», p. 272a.

112 Critique ironique de la poésie de paysage, Pei Ziye composa au moins deux poèmes sur la neige (sur les trois seuls conservés; cf. Lu Qinli, Xian Qin Han Wei Nanbei chao shi [Poèmes des époques antérieures aux Qin, des Han, Wei, Jin et Dynasties du Nord et du Sud], Pékin, Zhonghua shuju, 1983, p. 1790), dont l'un figure d'ailleurs dans le Yutai xinyong (Mu Kehong, Yutai xinyong jianzhu, Pékin, Zhonghua shuju, 1985, juan 8, p. 380).

113 Tongdian, juan 16, p. 389. 
Bien qu'il fasse référence au jugement de Yang Xiong sur le fu, Pei Ziye n'emploie pas dans ces lignes la métaphore des vermisseaux ciselés. Elle se trouve néanmoins dans le développement de Du You qui précède le texte dans le Tongdian - et non loin de là dans le mémoire de Shen Yue cité plus haut. Il est probable que Pei Ziye en usait dans une phrase que Du You n'a pas formellement incluse dans son «essai ». Néanmoins, il ne le faisait peut-être pas en tant que critique littéraire à proprement parler, mais en tant que juge de la manière dont les Liu Song sélectionnaient les talents. C'est en tout cas explicitement à ce titre que Du You inclut ce texte dans les « avis divers » ( « Zayi »), parmi les documents du Tongdian concernant le recrutement des officiers ( «Xuanju »), et non sans avoir d'abord rappelé quel fut l'essor de la poésie à la Cour et dans tout l'empire des Liu Song à partir de l'empereur Ming (r. 465-472), qui nommait ses ministres en fonction des poèmes composés lors de banquets.

Si Liu Xie choisit de porter les dragons ciselés au pinacle dans le titre de son traité au moment où Pei Ziye publiait le « Diaochong lun », où d'autres auteurs et puissants considéraient la gravivure de vermisseaux comme le comble de la futilité, du vice ou de la décadence, on suppose qu'il voulait d'autant mieux se démarquer de cette infamie. Le fait qu'il n'emploie jamais l'expression diaochong lorsqu' il condamne l'excès d'ornement en littérature et se contente de citer le jugement critique de Yang Xiong sur le $f u$ dans le chapitre qu'il consacre à ce genre ${ }^{114}$, semble conforter cette supposition. Il se peut fort bien que ses contemporains aient entendu résonner dans le titre du Wenxin diaolong comme un écho, par antithèse, des vermisseaux ciselés. À rebours des exercices de style et artifices littéraires, l'emblème de ce titre était une profession de foi en faveur du beau style : une sorte de proclamation de l'honneur et de la légitimité de l'ornement littéraire dès lors qu'il possède l'auguste prestige du dragon, la finesse et la beauté de son décor.

114 «Quanfu ( Examination de l'exposition), VIII, p. 307. 


\section{Variations sur un titre}

Il reste encore à reconnaître la fonction non pas symbolique ou polémique mais syntaxique de la métaphore du dragon ciselé dans le titre du Wenxin diaolong, à identifier le rapport qu'elle entretient avec l' «esprit de littérature ", son pendant ou complément (faussement) symétrique. Répondre à cette épineuse question - préliminaire à toute tentative de traduction - peut sembler présomptueux après que tant de savants s'y soient penchés 115 ; je tenterai seulement de démontrer ce pourquoi la solution que j'ai personnellement adoptée - et qui n'est aucunement originale puisqu'elle se contente de paraphraser celle de B. Alexéiev ( « Le cceur de la littérature en dragon sculpté ») ${ }^{116}$ — me paraît justifiée.

Pour quiconque s'est essayé à traduire le titre du Wenxin diaolong, il est au moins une évidence : les quatre caractères qui le composent peuvent a priori - si on les considère comme un ensemble syntaxique et non comme un titre - se lire de différentes manières : comme une phrase comportant un prédicat ( "l'esprit de littérature cisèle un dragon », ou « en gravant un dragon au cœur de la littérature $»){ }^{117}$, voire deux ( « orner l'esprit et graver

115 La politique de S. Owen dans ses remarquables Readings in Chinese Literary Thought, à savoir la romanisation du seul titre du Wenxin diaolong parmi les dix textes et ouvrages de critique litteraire dont il traite principalement, est peut-être la plus sage, même si elle est justifiée par une perplexité presque rageuse et sans doute injuste devant l'« explication remarquablement inefficace » (ibid., p. 185) donnée par Liu Xie de son titre dans la « Déclaration d'intention ».

116 Cf. La littérature chinoise. Six Conférences au Collège de France et au Musée Guimet (nov. 1926), Paris, P. Geuthner, 1937, p. 24.

117 Cf. A. Lévy, La littérature chinoise, Paris, PUF, Paris, 1991, p. 52 (« Que sais-je ») : Le creur de la littérature sculpte des dragons signifie que « les œuvres artistiques sont le produit de l'esprit littéraire». L'hypothèse « The Literary Mind Carves Dragons " est également envisagée par S. Owen (op. cit., p. 185). La solution de G. Yang et Yang Hsien-yi, Carving a Dragon at the Core of Literature, rend bien compte du caractère ludique qu'on peut trouver à ce titre, mais au prix d'un sacrifice important : le sens et la valeur du composé wenxin. 
un dragon ») ${ }^{118}$; comme deux groupes nominaux coordonnés («l'esprit de littérature et les dragons ciselés ») ${ }^{119}$ ou juxtaposés ( « l'esprit de littérature ou la gravure de dragons $»)^{120}$; comme un seul groupe nominal faisant se succéder un déterminant et un déterminé (le premier - wenxin

118 Je ne connais pas d'exemple de traduction selon cette lecture, mais la solution adoptée par G. Gagnon (« La postface personnelle de Liu Zhiji au Shitong. Un essai d'ego-histoire? », in J.-P. Drège (éd.), De Dunhuang au Japon, p. 362), Finesse de l'esprit, auvre d'esthétique, est la seule qui rende compte de l'éventuelle valeur prédicative de wen, valeur suggérée, parmi toutes les éventualités que permet le jeu poétique, par la symétrie avec le prédicat diao.

119 Cette solution qui porte l'immense mérite de la simplicité a été adoptée par de nombreux traducteurs occidentaux : V. Shih (The Literary Mind and the Carving of Dragons), J. Pimpaneau (Le cceur de la littérature et la sculpture des dragons, cf. La littérature chinoise, Arles/Paris, Philippe Picquier, 1989, p. 109), ou encore A. Relinque Eleta (El corazón de la literatura y el cincelado de dragones). D. A. Gibbs (Literary Theory in the Wen-hsin Tiao-lung, University of Washington, Ph.D., 1970, p. 85) en a donné une variante moins littérale : The Genesis and Artistry of Literature. On peut considérer la solution de E. R. Hughes, The Literary Mind and Its Carving of Dragons (The Art of Letters. Lu Chi's "Wen Fu ", A.D. 302, New York, Pantheon Books, 1951, p. 235), comme une variante supplémentaire, mais qui rend également compte de la lecture précédente ( The Literary Mind Carves dragons »).

120 Cf. Du Lijun («Lun Wenxin diaolong wenxue lilun siwei chengjiu » [À propos des apports du Wenxin diaolong à la théorie de la littérature], Rao Pengzi [dir.], Wenxin diaolong yanjiu huicui, p. 213): Literary Creation as Carving Dragons; P. Jacob (d'après A. Relinque Eleta, El corazón de la literatura y el cincelado de dragones, p. 14, n. 5) : De l'esprit dans les lettres ou la gravure des dragons ; J.J.Y. Liu (Chinese Theories of Literature, Chicago/Londres, University of Chicago Press, 1975, p. 21) : The Literary Mind : Elaborations ; ou A. Lavagnino: Il Tesoro delle lettere : un intaglio di draghi. Les deux premières solutions supposent une double définition - par analogie ou par équivalence - du sujet du traité ; les deux dernières en distinguent le sujet (wenxin) et la forme (diaolong). 
—étant subordonné au second — diaolong — : « le dragon ciselé de l'esprit de littérature » ${ }^{121}$ ou « dragons ciselés sur l'esprit de littérature » ${ }^{122}$ ).

Même si toutes les autres solutions doivent être prises en compte dans le large éventail des significations suggérées par la façon dont les quatre mots du titre du Wenxin diaolong jouent effectivement les uns avec les autres — bien au-delà du simple jeu de mots et en vertu d'une fonction très poétique du langage —, la dernière est celle qui se justifie le mieux s'agissant d'un titre. Si séduisante que soit l'association de l'esprit (ou essence) avec l'ornement du dragon - en un mot, du fond et de la forme - dans une paire de thèmes complémentaires, il ne semble pas juste de traduire ce titre sur le modèle de la simple coordination. J.R. Hightower ${ }^{123}$ fut le premier savant occidental à démontrer le fait, en proposant cette traduction (non définitive): «A Serious and Elegant Treatise on the Art/Secret of

121 Selon la valeur donnée à chacun des deux composés, cette lecture aboutit à des traductions divergentes. Plusieurs traducteurs font de l'esprit de littérature celui de l'auteur du traité, à l'instar de J.J.Y. Liu (Dragon Carvings of a Literary Mind, cf. The Art of Chinese Poetry, Chicago/Londres, University of Chicago Press, 1962, p. 71) avant qu'il n'adopte la solution citée dans la note précédente, ou de F.W. Cleaves (Carved Dragon of a Literary Heart, cf. "The Academician V.M. Alexeev as a Historian of Chinese Literature», traduction d'un article en russe de L.Z. Ejdlin [1946], HJAS, X [1947], p. 51). D' autres en font le sujet du traité : cf. par exemple Chen Shih-hsiang (Anatomy of the Literary Mind, cf. "In Search of the Beginning of Chinese Literary Criticism », Semitic and Oriental Studies, Berkeley/Los Angeles, University of California Publications in Semitic Philology [vol. 11], 1951, p. 57), ou, dans une version plus litterale, Chen Shouyi (The Carved Dragon of the Literary Mind, d'après J.J.Y. Liu, Chinese Theories of Literature, p. 146, note 24). Je ne résiste pas au plaisir de citer la proposition plus libre de Chen Shouyi, Secrets to Literary Success, qui à maints égards rend parfaitement compte du propos de Liu Xie.

122 Cf. par exemple W.C. Fisk : Elaborate Carvings on the Literary Mind (Formal Themes in Medieval Chinese and Modern Western Literary Theory. Mimesis, Intertextuality, Figurativeness, and Foregrounding, Ph.D., Madison, University of Wisconsin, 1976, p. 12).

123 Compte rendu de la traduction du Wenxin diaolong par V. Shih, HJAS, 22, 1959 , p. 286. 
Literature », et J.J.Y. Liu expliqua clairement : «Il est amplement évident que wenxin renvoie au sujet du livre, tandis que diaolong renvoie à sa nature ; les deux expressions ne peuvent pas être prises comme les deux principaux sujets de l'ouvrage. Du point de vue de la syntaxe, les titres des cuvres chinoises anciennes prennent généralement la forme "verbe plus objet" (par exemple, Lunwen, "en discutant de la littérature"), ou "nom plus nom", le premier nom exposant le sujet et le second décrivant sa nature (par exemple, Wen fu, "une exposition sur la littérature"). » 124

On ne connaît en effet aucun titre chinois ancien où soient associés deux termes définissant strictement le sujet de l'ouvrage sans en annoncer également la forme, la manière ou l'ambition ${ }^{125}$. On peut développer l'affirmation de J.J.Y. Liu en citant quelques titres tétrasyllabiques pour la plupart fort célèbres : Shuowen jiezi (Explication des caractères simples et analyse des caractères composés) redouble la structure « verbe-objet »; Shishuo xinyu (Nouveaux propos racontés dans le monde) et Yutai xinyong (Nouveaux chants de la terrasse de jade) associent un déterminant à un déterminé qui désigne la forme ou nature de l'ouvrage ; Fengsu tongyi (Signification générale des coutumes et des mœurs) et Zhengshi xiaofan ${ }^{126}$ (Simplification - ou abrégé - de l'histoire officielle) en affichent de la même manière l'ambition ou la méthode. Dans les Chunqiu fanlu (Rosée abondante - ou couronne - des Printemps et Automnes), Wenzhang yinghua ${ }^{127}$ (Florilège des belles-lettres) ou Fayuan zhulin (Forêt des perles

124 Chinese Theories of Literature, p. 146-147, note 24.

125 Parmi les structures les moins frequentes, on trouve des titres désignant le seul contenu d'un recueil, tels que Yudui ou Yuli (Propos parallèles, cf. Sui shu, juan 34, p. 1008) ; utilisant une métaphore sans que soit énoncé explicitement le sujet ou contexte du recueil, tel que le Caibi (Jades choisis, cf. ibid., p. 1007) de Yu Jianwu (490-552?); ou encore un titre enonçant la fonction, et même le destinataire de l'ouvrage, Tongwu (Pour la compréhension des enfants, Manuel d'histoire pour enfants en douze juan ; cf. Sui shu, juan, 33, p. 961).

126 Ouvrage recensé par le Sui shu (juan 33, p. 961) comme ayant compté quatrevingt-quatorze juan, de la main de Ruan Xiaoxu (479-536), l'auteur du catalogue bibliographique Qilu (Sept Registres).

127 Anthologie de poèmes pentasyllabiques compilée sous la direction de Xiao 
du jardin du dharma, de Daoshi [?-683]), c'est une métaphore qui sert à désigner la nature ou l'ambition de l'ouvrage : le titre du Wenxin diaolong obéit à ce dernier modèle.

À l'argument syntaxique on peut encore ajouter celui des usages observés par Liu Xie lui-même et par ses préfaciers ou lecteurs postérieurs lorsqu'ils emploient une forme abrégée du titre du Wenxin diaolong. Le premier usage en cette matière n'est pas le plus souvent représenté, mais il est celui que respecte Liu Xie quand il introduit, dans la «Déclaration d'intention », son guide de lecture du traité : «L'Esprit de littérature est ainsi composé qu'il prend le dao pour origine... » ${ }^{128}$ Liu Zhiji (661-721) s'y conforme dans la «Postface personnelle » du Shitong (Traité de l'historien parfait) ${ }^{129}$, ainsi que plusieurs préfaciers du Wenxin diaolong sous les Ming et les Qing : Cheng Kuan (d.i.) explique par exemple «ce pourquoi l'Esprit de littérature de maître Liu est un dragon ciselé » ( $c i$ Liuzi Wenxin zhi suoyi wei diaolong ye), et désigne par ailleurs à trois reprises cet «Esprit de littérature » ${ }^{130}$.

À l'inverse, vers 610, Liu Shanjing (d. i.) citait le Wenxin diaolong comme « ouvrage en Dragon ciselé »131. Wang Yinglin (1223-1296) introduisit une citation du chapitre « Mingshi »(VI : Illustration du poème) par les mots

Tong en vingt juan selon sa biographie dans le Liang shu (juan 8, p. 171) et trente selon le traité bibliographique du Sui shu (juan 35, p. 1084); elle n'a pas été conservée.

128 «Xuzhi », L, p. 1924.

129 Cf. «Zixu », chapitre XXXVI ; G. Gagnon, Concordance combinée du Shitong $e t d u$ Shitong xiaofan, Paris, Collège de France, Institut des Hautes Études Chinoises, 1977, vol. 1, segment 7743.

130 Préface à l'édition du Wenxin diaolong de 1540 annotée par Xu Bo, reproduite par Wang Liqi, op. cit., p. 141-2 ; p. 141. Dans la préface de sa copie manuscrite incomplète du Wenxin diaolong, Jin Xuan (XVII siècle) se réfère à « L'Esprit de littérature composé par [Liu] Xie en dix fascicules » (cf. Wang Liqi, op. cit., p. 149). Zhang Xuecheng désigne l'ouvrage de la même manière (cf. Wenshi tongyi, « Neipian », juan 5, « Shihua », p. 559).

131 D'après le Bunkyô hifuron (Miroir des lettres du magasin des secrets ; Wang Liqi, Bunkyô hifuron jiaozhu, Pékin, Zhongguo shehui kexue chubanshe, 1983) du moine japonais Kûkai (Kôbô daishi, 774-835), "Tian », "Sisheng lun », p. 90 . 


\section{Valérie Lavoix}

« le Dragon ciselé dit [...] ${ }^{132}$. Sous les Ming, le préfacier Gu Qiyuan ${ }^{133}$ écrivait que Liu Xie « a donné pour titre à son propre [ouvrage] le Dragon ciselé », et Cao Xuequan donnait une intéressante explication du fait que le Wenxin diaolong n'est pas mentionné dans le Wenxian tongkao (Étude critique générale des textes et documents) de Ma Duanlin (1254 ?-1323) :

Les savants des Song détestaient tout particulièrement le mot ornement (wen). Comme s'y ajoutait de surcrốt l'appellation (hao) « dragon ciselé », leur regard pouvait d'autant moins s'arrêter sur cet ouvrage. ${ }^{134}$

Pour clore son « Fu sur le Wenxin diaolong de Liu Yanhe », Li Zhizhong (d.i.) se livra à une dernière variation sur le titre du traité :

Magique est la créature qu'est le dragon, bien achevé est le [sens du] mot "ciselé »; un tel esprit pouvait seul dénombrer les littérateurs des âges passés. ${ }^{135}$

Enfin, selon Zhang Songsun (d.i.):

[Liu Xie] sculpta un style bien orné, comme s'il faisait naître le phénix pour s'envoler ; par le détour subtil d'une énigme, il choisit pour appellation le dragon ciselé. ${ }^{136}$

Les auteurs qui se réfèrent au Wenxin diaolong par les seuls mots « dragon ciselé » font de cette expression l'appellation formelle du traité et rendent hommage au coup de génie de Liu Xie en acceptant la valeur dénominative, ou titulaire, de la métaphore. Ceux qui comme son auteur

132 Kunxue jiwen (Registre des connaissances acquises par une étude assidue), « Pingshi », d'après Wang Liqi, Wenxin diaolong xinshu, p. 157.

133 Préface datée de 1609, reproduite dans ibid., p. 147.

134 Préface au Wenxin diaolong, p. 148 (cf. ci-dessus, note 40). Cao Xuequan emploie à trois reprises la forme abrégée Diaolong dans cette préface. $\mathrm{Cf}$. également les textes cités par Wang Liqi, ibid., p. 159-160, 162, 164.

135 «Liu Yanhe Wenxin diaolong fu », reproduit dans ibid., p. 167.

136 Préface à l'édition du Wenxin diaolong datée de 1791, reproduite dans ibid., p. 151. 
évoquent au contraire l'Esprit de littérature privilégient sans doute très naturellement le sujet de l'ouvrage par rapport à sa forme - le contenu plutôt que le contenant - ; on imagine mal, en revanche, qu'on ait pu choisir les «dragons ciselés » plutôt que l' « esprit de littérature » comme titre abrégé, si l'une et l'autre expressions avaient énoncé les deux sujets du traité, coordonnés sur le même plan et avec la même valeur.

On ne sait si Liu Xie fut bien le premier (et le dernier) à employer la métaphore du dragon ciselé - ordinairement l'art des œuvres bien composées ou le talent du beau style — pour désigner le « genre » de son ouvrage, à l'instar des « jardins », « forêts », « océans », « florilèges », « miroir universel » et autres « rosées abondantes » de la littérature ancienne. Certains de ses lecteurs ont pu le trouver bien orgueilleux de prétendre avoir composé un ouvrage si beau, mais l'expression était à son époque à la fois juste assez laudative pour évincer les critiques des vermisseaux ciselés et suffisamment banalisée pour servir de terme générique et technique. Elle lui permettait assurément de proclamer sa double ambition de littérateur et de critique : l'architecture parfaitement accomplie de son traité et la beauté très raffinée de sa prose ne démentent pas l'emblème du titre du Wenxin diaolong.

Quoi qu'il en soit, si Liu Xie se conformait bien aux usages du passé en matière de titres (du point de vue syntaxique) en décrétant que son ouvrage était un «dragon ciselé sur - ou pour — l'esprit de littérature », il ne se privait sans doute pas de jouer simultanément des autres lectures possibles des quatre mots qu'il avait assemblés. J'en vois une preuve dans le jeu non plus poétique mais graphique - graphiquement poétique au sens où l'écriture chinoise le permet - qui donne en vérité la toute première glose du titre du Wenxin diaolong : la disparité et la complémentarité parfaite des deux paires de caractères associées, l'une désignant la substance même par la simplicité épurée, indécomposable de wen et de xin, l'autre une forme très construite, ornementée et magnifiquement équilibrée comme diao et long. Pour cette raison, il me semble dommage de renoncer à traduire littéralement ce titre, bien que le "dragon ciselé » soit pour le lecteur occidental une énigme trop exotique et bien plus mystérieuse qu'elle ne l'est en chinois. Enfin, la formulation choisie par B. Aléxeiev - Le Cour de la littérature en dragon sculpté - étant celle qui préserve le mieux la 


\section{Valérie Lavoix}

possibilité du jeu poétique, et notamment le balancement rythmique et paradoxal entre l' « esprit » et le «dragon », la trace d'un parallèle entre l'art littéraire et la gravure, qu'elle soit plagiée en guise d'ultime variation : l'Esprit de littérature en dragon ciselé.

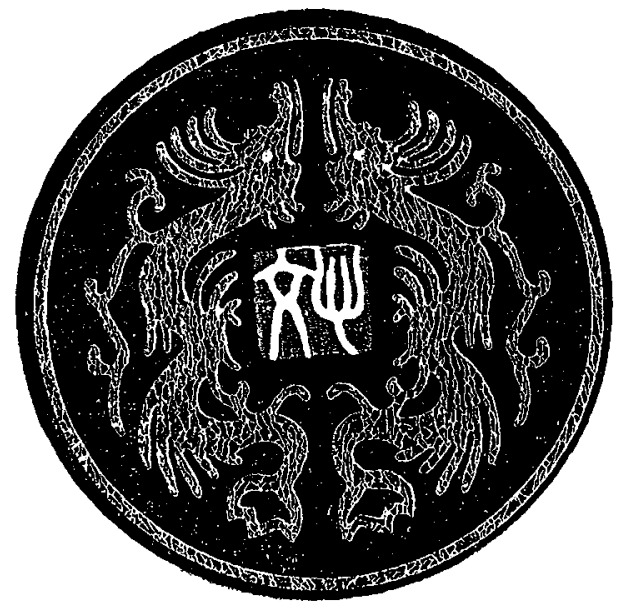




\section{Principaux caractères chinois}

Apitan xin lun 阿毗譬心論

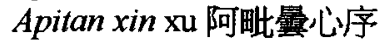
Anping 安平

$\mathrm{Ban} \mathrm{Gu}$ 班固

bian 辯

Bie fu 別賦

Bielu 別錄

Bohai 勃海

boyi 博依

Bunkyô hifuron 文鏡秘府論

Caibi 探璧

Cai Yong 蔡畠

Cao Pi 曹丕

Cao Xuequan 曹學佺

Cao Zhi 曹植

Chen Lin 陳琳

Cheng Kuan 程寬

chongshu 蟲書

Chu Sanzang ji ji 出三藏記集

Chunqiu fanlu 春秋繁露

Chunyu Kun 渖于髧

ci Liuzi Wenxin zhi suoyi wei diaolong ye 此劉子文心之 所以爲雕龍也

Cui Lie 崔烈

Cui Shi 崔寔

Cui Yin 崔馹

Cui Yuan 崔瑗

Daming 大明

Daren fu 大人賦

Daren zhi song 大人之頌

Da Wei taizi jian 答魏太子侥

Daxu 大序

Daoshi 道世

Dianliue 典略

diao 雕 (䧓)

diaochong 雕蟲

diaochong boji 雕蟲薄伎(技)
Diaochong ji 雕蟲集

Diaochong lun 雕蛞論

diaochong xiaodao 雕蟲小道

diaochong xiaoji 雕蟲小伎 (技)

diaochong xiaoyi 雕蟲小藝

diaochong zhuanke 雕蟲策刻

diaolong 雕龍

Diao Wei Wudi wen 韦魏武帝文

Dinglinsi 定林寺

Donghun hou 東昏侯

Dong Zhongshu 董仲舒

Du Fu 杜甫

Du You 杜佑

dunxin 䁖心

Fayan 法言

Fayuan zhulin 法范珠林

Fan Ye 范瞱 (Weizong 蔚宗)

Fan Yun 范雲

fei zhi hu liyi 非止乎禮義

feng 風(諷)

Fengsu tongyi 風俗通義

$\mathrm{Gan} \mathrm{Bao}$ 干寶

Gao Yun 高允

gongti 宮體

Guji liezhuan 滑稽列傳

Gu Qiyuan 顧起元

hao 號

hao cifu 好辭賦

hao weiwenzhang 好爲文章

$\mathrm{He}$ 和

Hongming $j i$ 弓明集

houjin zhi shi 後進之士

Huan 環

Huangfu Mi 皇甫㖹

Huiyuan 慧遠

Jitian fu 藉田賦

Jixia 稷下

Jian'an qizi 建安七子 
Jiankang shilu 建康寶錄

Jiang Yan 江淹

Jie 架

Jin Xuan 謹軒

Jingshan 荆山

$\mathrm{Juan}^{a}$ 涓

$J^{J u a n}{ }^{b}$ 蜎

Juanzi ${ }^{a}$ 涓子

$J_{u a n z i}^{b}$ 蜎子

kefu 刻符

Kôbô daishi 弘法大師

Kûkai 空海

Li E 李摆

Li Fang 李昉

Li Shan 李善

Liyun 裋運

Li Zhizhong 李執中

Liang 梁

Liang Wudi ji xu 梁武帝集序

Liexian zhuan 列仙傳

Ling 哃

Liu Bao 劉苞

Liu Hui 劉繪

Liu Ji 劉洎

Liu Xiaoyi 劉孝儀 (Qian 潛)

Liu Ru 劉需

Liu Shanjing 劉善經

Liu Xiang 劉向

Liu Xiaochuo 劉孝綽

Liu Xiaowei 劉孝威

Liu Xiaoyi 劉孝儀

Liu Xie 劉努 (Yanhe 彥和)

Liu Yanhe Wenxin diaolong fu 劉彥和文心雕龍賦

Liu Zhiji 劉知幾

Liu Zun 劉要

longwen 龍文

$\mathrm{Lu} \mathrm{Ji}$ 陸機

Lu Jue 陸厥

Lushan 盧山 lun 論

Lunshui 論說

Lunwen 論文

Ma Duanlin 馬端臨

Miaode xiansheng zhuan

妙德先生傳

Mingshi 明詩

Mou 牟

Pan Yue 潘岳

Pei Songzhi 裴松之

Pei Yin 裴䮮

Pei Ziye 裴子野

Pengcheng 彭城

pianshi 篇什

Pingshi 評詩

qi 豈

Qi ao 淇奧

Qilu 七錄

Qiaoxin 巧心

Qiao Xuan 喬(橋) 玄

Qinxin 琴心

Quanfu 詮賦

Rangwang 讓王

Ren Fang 任昉

Rongcai 鎔裁

Runan 汝南

Ruan Ji 阮籍

Ruan Xiaoxu 阮孝緒

Sandu fu xu 三都賦序

se 色

Sengyou 僧祐

Shensi 神思

Shen Yue 沈約

Shipin 詩品

shi shan diaolong 世禪雕龍

Shishuo xinyu 世說新語

Shixu 時序

Shixun 釋訓

Shuowen jiezi 說文解字

Sima Qian 司馬遷 
Sima Xiangru 司馬相如 (Zhangqing 長卿)

Sisheng lun 四聲論

Sixun song 四巡頌

Song liee 宋略

Song Yu 宋玉

Soushen ji 搜神記

Su Liang 蘇亮

Sui (hou) 随 (侯)

Taiwei Qiao Xuan beiyin 太尉喬玄碑陰

Taiwu 太武

Tian 天

Tiandao 天道

Tianren jing 天人經

Tongdian 通典

Tongwu 童悟

Wang Can 王聚

Wang Jian 王儉

Wang Jun 王筑

Wangsunzi 王係子

Wang Wei 王維

Wang Yinglin 王應䊁

Wang Zhen 王貞

Wang Zhu 王助

Wei 魏

Wei Fan shangshu rang libu fenghou diyi biao 爲范尚書 讓吏部封侯第一表

Weifeng 衛風

weiwen 爲文

weiwen yong 爲文用

weiwenzhang 爲文章

weiwen zhi yongxin 爲文之用心

wen bing diaolong 文炳雕龍

wenci 文辭

Wende 文德

wen fan diaolong 文繁雕龍

Wen fu 文賦

Wenhui 文惠 wen shan diaolong 文擅雕龍

Wenshi tongyi 文史通義

Wenxian tongkao 文獻通考

wenxin 文心

Wenxin diaolong 文心雕龍

Wenxuan 文選

Wenyan 文言

Wenyuan liezhuan 文苑列傳

Wenyuan yinghua 文苑英華

wenzhang 文章

Wenzhang yinghua 文章英華

wen zhao diaolong 文昭雕龍

Wubifa xin lun 無比法心論

wu jixin zhi suo 無寄心之所

Wu Zhi 吳質

Wuzi 吾子

$\mathrm{Xici}$ 熬鳏

xi zhi weiwenzhe 昔之爲文者

Xiangdong 湘東

Xiao Gang 開綱 (Jianwen 簡文)

xiaolian 孝廉

Xiaoming 孝明

Xiao Tong 莘統 (Zhaoming taizi 照明太子)

Xiao Yan 萧行

Xiao Yaoguang 蕀遙光

(Shi' an 始安)

Xie Tiao 謝朓

Xie weiwen chang yu foli

就爲文長於佛理

xinli 心力

Xing Shao 邢邵 (劭)

xiucai 秀才

Xu Bo 徐渤

Xu Song 許嵩

Xuzhi 序志

Xuande huanghou 宣德皇后

Xuande huanghou ling

宣德皇后令

Xuanju 選舉 


\author{
Xue Daoheng 薛道衡 \\ Xueji 學記 \\ Yan Yanzhi 顏延之 \\ yan zhi wen 言之文 \\ Yang Xiong 揚雄 (Ziyun 子雲) \\ Yang Xiu 楊脩 \\ Ye Lianfang 葉聯芳 \\ yici 遺辭 \\ yinyong qingxing 吟詠情性 \\ Ying 應 \\ yongxin 用心 \\ yongxin yu wen 用心於文 \\ $Y u d u i$ 語對 \\ Yu Jianwu 庽肩吾 \\ Yuli 哣麗

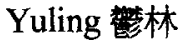 \\ Yutai xinyong 王臺新詠 \\ Yu Xiangdong wang shu \\ 與湘東王書 \\ Yu Yang Dezu shu 與楊德祖書 \\ Yuan 淵 \\ Yuan Can 袁聚 \\ Yuandao 原道 \\ Yuanjia 元嘉
}

\author{
Zayi 雜議 \\ zan 䁈 (謴) \\ Zhang Heng 張衡 \\ Zhang Songsun 張松孫 \\ Zhang Xuansu 張玄素 \\ Zhang Xuecheng 章學誠 \\ Zhengshi song 徵士頌 \\ Zhengshi xiaofan 正史削繁 \\ zhi 筫 \\ Zhong Rong 鍾嶸 \\ Zhou 紂 \\ zhuanke weiwen 䇡刻后文 \\ zhuo yu weiwen 拙於爲文 \\ Zixia 子夏 \\ Zixu 自敘 \\ Ziyou 子游 \\ Zongshu 總術

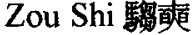 \\ Zoushizi 騮車豖子 \\ Zou Yan 騮行 \\ Zouzi 龮子 \\ Zouzi zhongshi 騽子終始 \\ Zuo Si 左思
}




\title{
Variations sur le titre du Wenxin diaolong
}

\section{Résumé}

Valérie LAvoIx : Un dragon pour emblème. Variations sur le titre du Wenxin diaolong

Le titre du plus célèbre traité de critique littéraire chinois, le Wenxin diaolong de Liu Xie ( $c a$ 465-ca 521), a été interprété de diverses manières et résiste âprement à la traduction. Il associe deux composés dont le premier est totalement inédit, et le second hérite au contraire d'une assez longue histoire en tant que métaphore et stéréotype. À la fois précisément défini par l'auteur et signalé comme polysémique, I' « esprit de littérature » (wenxin) place au pinacle de l'œuvre, et au centre du discours sur la littérature, la notion de «cceur/esprit »- moteur et finalité de toute création littéraire. Quant au «dragon ciselé » (diaolong), il proclame la glorieuse légitimité du beau style contre l'infamie des vermisseaux ciselés (diaochong) fustigés au même moment par Pei Ziye (469-530), tout en affichant l'ambition littéraire de l'auteur. Car l'invention consiste ici à solliciter la métaphore pour lui faire désigner la nature même de l'ouvrage, ainsi que le comprennent quasi unanimement ses commentateurs et préfaciers. Parmi toutes les lectures potentiellement ouvertes de ce titre, la disparité graphique de ses quatre caractères souligne parfaitement le parallélisme formel, subtilement faussé, qui le constitue.

\begin{abstract}
Valérie LAvoIX: A Dragon for Emblem. Variations on the Title of the Wenxin diaolong

The title of the most famous treatise of Chinese literary criticism, Liu Xie's (ca 465-ca 521) Wenxin diaolong, was interpreted in different manners and is a challenge to translation. It combines two word-compounds, the first of which is unprecedented, while the second has enjoyed a relatively long history as metaphor and stereotype. Both precisely defined by the author and identified as polysemic, the "mind of literature" (wenxin) places the notion of "heart/mind" - the drive and end of any literary creation - at the pinacle of the work and at the centre of the discourse on literature. As for the "chiselled dragon" (diaolong), it proclaims the glorious legitimacy of beautiful style, against the infamy of the "chiselled worms" (diaochong) fustigated at the same period by Pei Ziye (469-530), while demonstrating the author's literary ambition. The invention consists here in seeking the metaphor of the «chiselled dragon » to identify the nature of the work, as is understood almost unanimously by commentators and preface writers. Among all the possible readings of this title, the graphic disparity of its four characters underlines perfectly the formal parallelism, acutely falsified, which characterizes it.
\end{abstract}

